Historic, archived document

Do not assume content reflects current scientific knowledge, policies, or practices. 


\section{WILDERNESS UUSE ESTMMATION: \\ A PILOT TEST OF SAMPLIJNG PROCLDVURES \\ ON WHE 15 P 8:08}

MISSION MOURTARISS PRIMTTIVE AREA

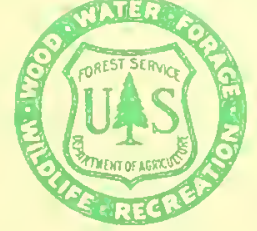

USDA Forest Service Research Paper INT-109, 1971

INTERMOUNTAIN FOREST AND RANGE

EXPERIMENT STATION

Ogden, Utah 84401 


\section{THE AUTHORS}

Dr. Robert C. Lucas is Principal Geographer and Project Leader for Wilderness Management Research, Intermountain Forest and Range Experiment Station, Missoula, Montana. Dr. Hans T. Schreuder is Mathematical Statistician, Southeastern Forest Experiment Station, Research Triangle Park, North Carolina. George A. James is Principal Recreation Specialist and Project Leader for Forest Recreation Inventory and Management Research, Southeastern Forest Experiment Station, Asheville, North Carolina. The cooperation of the staff of the Flathead National Forest, and particularly those serving on the Condon Ranger District, is gratefully acknowledged.

Cover Photo: Spectacular view of north face of Gray Wolf Peak that rises abruptly above southwest shore of Gray Wolf Lake. St. Mary's Peak in center. A 12-year-old mountain climber in foreground. 


\title{
WILDERNESS USE ESTIMATION: A PILOT TEST OF SAMPLING PROCEDURES ON THE MISSION MOUNTAINS PRIMITIVE AREA
}

\author{
Robert C. Lucas, Hans T. Schreuder, and George A. James
}

INTERMOUNTAIN FOREST AND RANGE EXPERIMENT STATION Forest Service

U.S. Department of Agriculture

Ogden, Utah 84401

Robert W. Harris, Director 


\begin{abstract}
Methods for estimating wilderness recreational use were pilot tested during the summer and fall of 1968 in the Mission Mountains Primitive Area. The main basis for the estimating methods was trail register information. Intensive field sampling provided data on actual use to relate to registration data. Both ratio and regression methods produced estimates with acceptably small error terms (6 percent for estimated visits, in the case of the ratio estimates, for example), but the ratio methods seemed to have a small advantage over regression methods. Overall, 65 percent of the visitor groups were estimated to have registered, but there were substantial variations in registration rates associated with a number of factors--type of activity, mode of travel, length of stay, and weather. The registration rate patterns were generally similar to those reported from an earlier study in Oregon. Use of the Mission Mountains Primitive Area is described. Suggestions are made for wilderness use estimation and for increasing the effectiveness of trail register systems.
\end{abstract}




\section{CONTENTS}

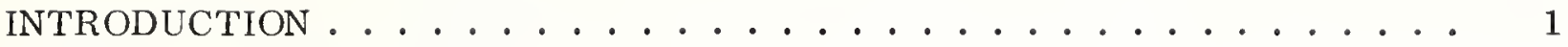

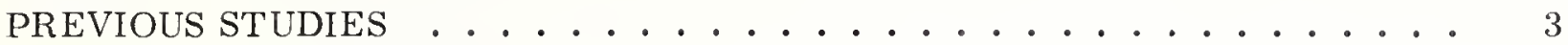

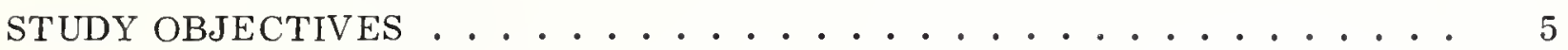

The Study Area ........................ 6

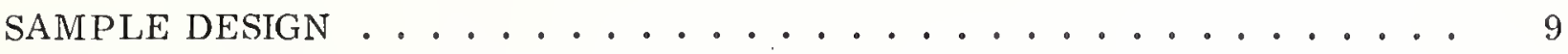

Sample Selection ....................... 10

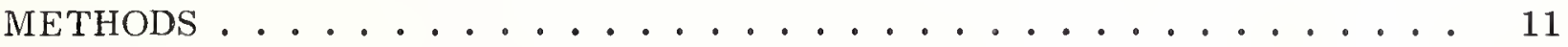

Interviewing Procedures ................... 11

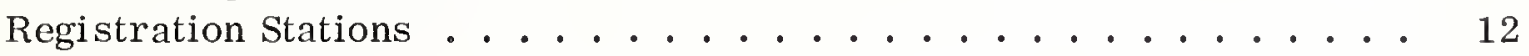

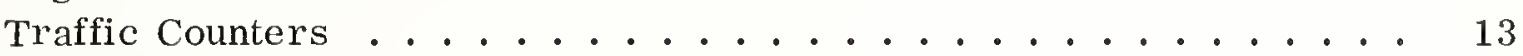

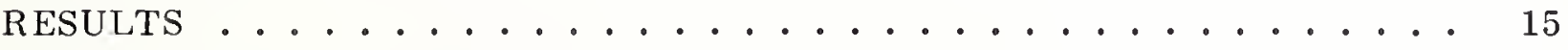

Registration Rates ..................... 15

Completeness of Response on Registration Cards . . . . . . . . 17

Accuracy of Planned Length of Stay .............. 18

Relationships of Visitor Characteristics, Other Variables to Rates . . 18

Reasons for Not Registering ............... 20

Comparison With Results of Previous Studies .......... 21

GENERATION OF USE ESTIMATES . . . . . . . . . . . 23

Registration Station Data .............. 24

Ratio Estimation (Corrected Registration Information) . . . . . . 25

Stratified Random-Sampling Estimation ............ 26

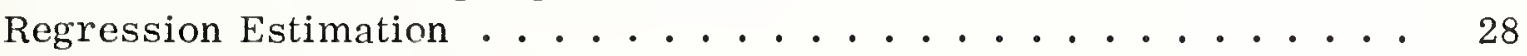

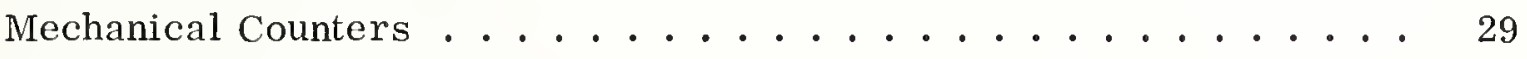

Summary of Use Estimates . . . . . . . . . . . 31

Cost of Using Same Estimation Technique . . . . . . . . 33

DISCUSSION $/$ RECOMMENDATIONS . . . . . . . . . . . 35

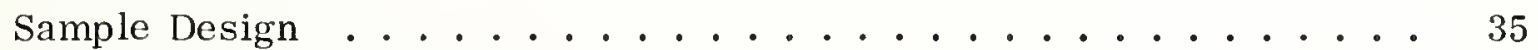

Best Estimation Procedures ................ 36

Extension of Results to Other Wildernesses . . . . . . . . 36

Personal Interviews and Automatic Counters .......... 36

Raising Registration Rates ................ 37

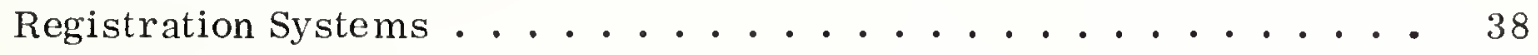

LITERATURE CITED . . . . . . . . . . . . . . . . 39

APPENDIX I--Primitive Area Registration Card . . . . . . . . . 41

APPENDIX II--Formula Used to Calculate Standard Errors for Estimated Registration Rates . . . . . . . . . 43

A PPENDIX III--Variables and Notation Used in Study . . . . . . . . . . 44 



\section{INTRODUCTION}

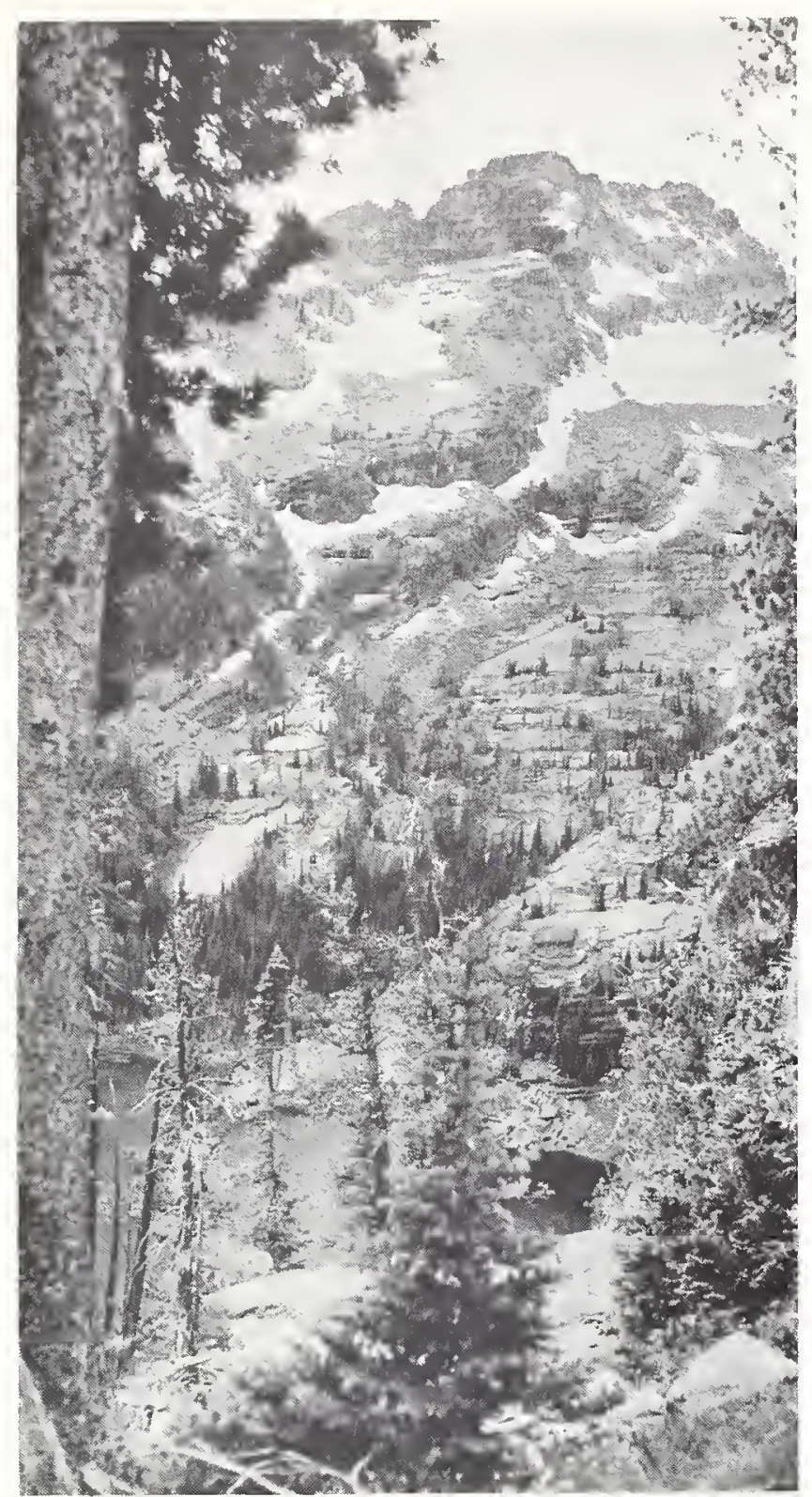

The rugged north face of Gray Wolf Peak as viewed from across the head of Gray Wolf Lake.

Wilderness is a fragile resource in both an ecological and an esthetic sense. Management of wilderness faces the difficult challenge of providing for the use of these areas while preserving the substantially unmodified ecosystem and the outstanding opportunities for solitude that are the essential characteristics of wilderness.

The impact of use on the wilderness resource cannot be understood or managed adequately without knowledge about the level and nature of that use. Many decisions need to be based on use data, for example, control of use by people and their horses, redistribution of use pressures, trail construction and maintenance, litter cleanup, campsite closures and rehabilitation, planning patrol routes for Wilderness Rangers, and minimizing conflicts between grazing and recreational use. Such decisions are hampered by the poor quality of wilderness recreation use estimates--among the poorest of recreation use data.

It is difficult to estimate wilderness use with even modest accuracy for obvious reasons. Wilderness visitors are highly mobile and are scattered widely over large areas, away from roads where observation and counting are easy. Users may enter a wilderness at many access points; several wildernesses have over 60 entrances. 
Some wildernesses have no system of visitor registration; managers of most of the areas that do have a registration system are uncertain how registered use compares with actual use. This is a serious problem, particularly because of the rapid growth of use of National Forest and National Park Wilderness. National Forest visitor-days of wilderness use were estimated at only one-twentieth of developed campground use in 1946, but 20 years later this proportion had grown to about one-seventh. We should be skeptical about the figures, but the trend is undeniably strongly upwards. Use projections strongly suggest large future increases. ${ }^{1}$

This rising use is occurring on a land base of established wilderness that has remained almost unchanged in total area during the past 30 years; thus, these growing use pressures need to be managed. Use estimates in the past that have been based largely on informal observation, experience, and intuition are not acceptable today. Consequently, the need for sound estimates of wilderness use now is critical--before wilderness values are irrevocably damaged or destroyed.

IUniversity of California Wildland Research Center, Wilderness and recreation-a report on resources, values, and problems, ORRRC Study Report 3, Outdoor Recreation Resources Review Comm., Wash., D.C., 1962, pp. 235-236, and unpublished projections of the National Forest Recreation Survey (NFRS) indicate about an eight- to tenfold increase between 1959 and 2000. 


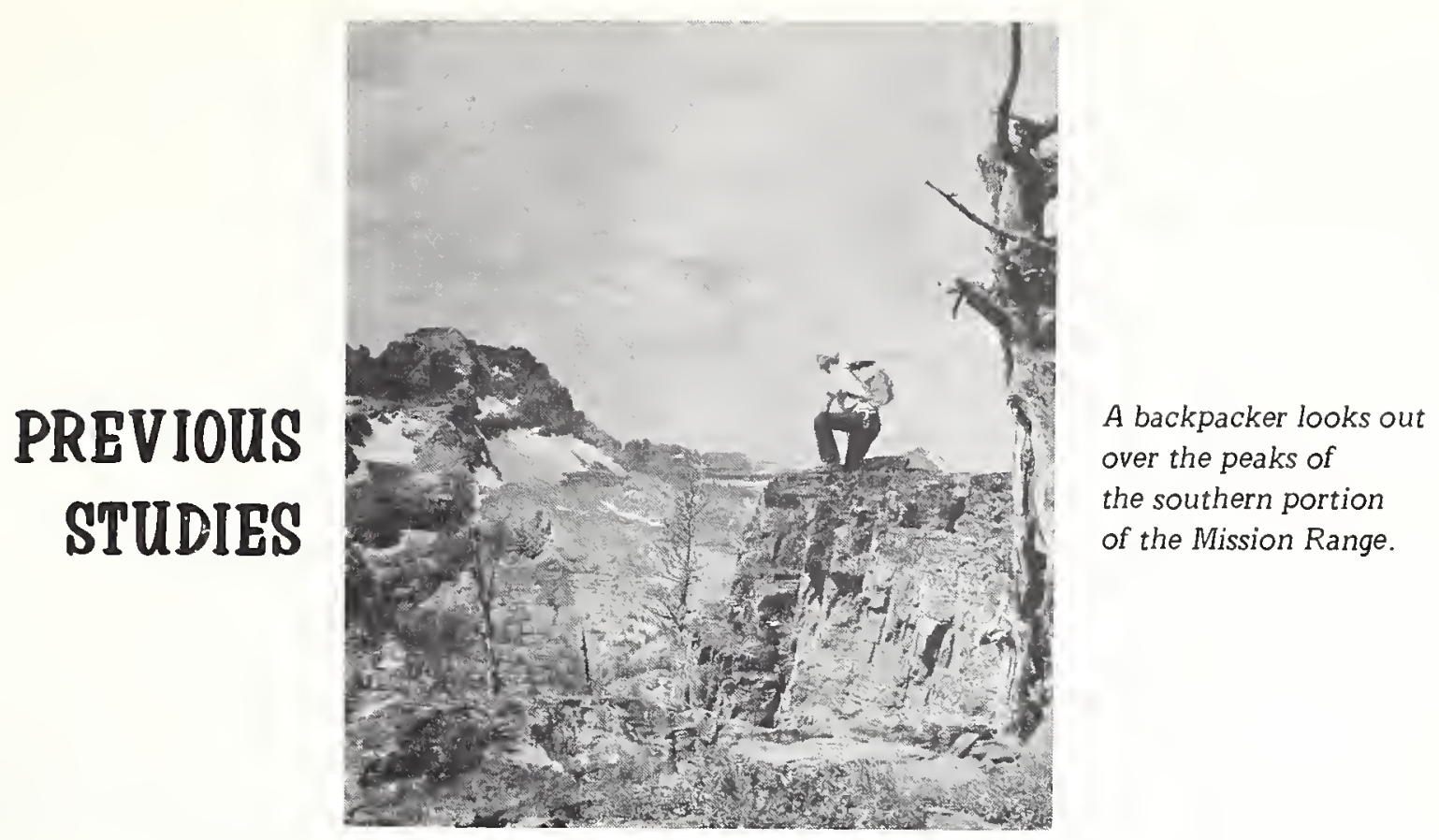

One of the first major efforts to develop ways of obtaining wilderness use information was made by Wenger and Gregersen in the Three Sisters and Mountain Lakes Wildernesses in Oregon during 1961 and 1962. Wenger (1964) tested the general effectiveness of unmanned, self-registration stations by placing two different types of registration boxes and forms and three different types of signs on trails and then interviewing visitors up the trails, out of sight of the registration stations. He concluded that self-registration was a promising approach to wilderness use estimations; that a single card per party, dropped into a box after completion, was superior to a book-type registration system; and that a firm but explanatory sign was best. Registration rates averaged 74 percent for both years and both areas. Location of registration stations had an important influence on response rates.

Wenger and Gregersen (1964) also reported on the association of registration rates and various visitor and location characteristics. They concluded that "raw" registration data contained biases, specifically underrepresenting riders, fishing and elkhunting parties, one-person parties, and repeat visitors. Many other factors--including trails, day of week, size of residence community and distance to it, length of stay, and occupation--were unrelated to registration rates. They also concluded that the effects of nonresponse must be fully considered, and that modifications must be made in findings stemming from raw registration data. Wenger and Gregersen did not report on the conversion of registration data into use estimates.

Lucas (1964) studied recreational use of the Quetico-Superior area in Minnesota and Ontario, Canada. Traffic counters were placed on six major access roads and drivers were briefly interviewed as they left the area. The sample included about 4,000 groups-approximately 4 percent of the total traffic. Estimates of recreational characteristics for the total measured traffic for each road were made on the basis of the composition of the sample interviews. Error terms were not calculated. Visits were shorter than expected; 41 percent of the visitors did not stay overnight, and the average visit was only 1.75 days. Use was also very unevenly distributed--10 percent of the access points accounted for over half of the total visitor use for every type of user. 
Thorsell (1968) studied wilderness visitor registration rates in Banff and Yoho National Parks in Canada. He observed six trail registration stations from a distance using binoculars without the knowledge of the visitors during a total of 95 hours. The stations were located at destinations in the interior of the Parks. On1y 35 percent of the visitors registered, a much lower rate than in Wenger's Oregon study. Thorsell attributes this to the long form he used, which contained 19 questions. He also reported great variation in registration rates between locations, but this could be due to the small sample at each location. He classified all registration stations into three rates of response, and multiplied registration totals by the inverse of the assumed rate of response in order to estimate use. A similar procedure, based on an arbitrarily assumed 75 percent registration rate, was used by Thorsell (1967) in an earlier study of Waterton Lakes National Park. He reported that about 14 percent of Banff visitors used the trails, but in a very uneven distribution; the most popular trail out of a total of 56 trails in the Park accounted for 39 percent of the trail visitors. Steepness of trails did not seem to affect amount of use. Day use predominated (94 percent). Only 11 percent of the trail visitors penetrated over 5 miles into the backcountry.

Cushwa et al. (1965), and James and Henley (1968) conducted pilot sampling studies to estimate dispersed recreation use on large, general recreation areas. Their technique incorporated interviewing recreationists at established road checkpoints as they left recreation areas in nonwilderness situations. 


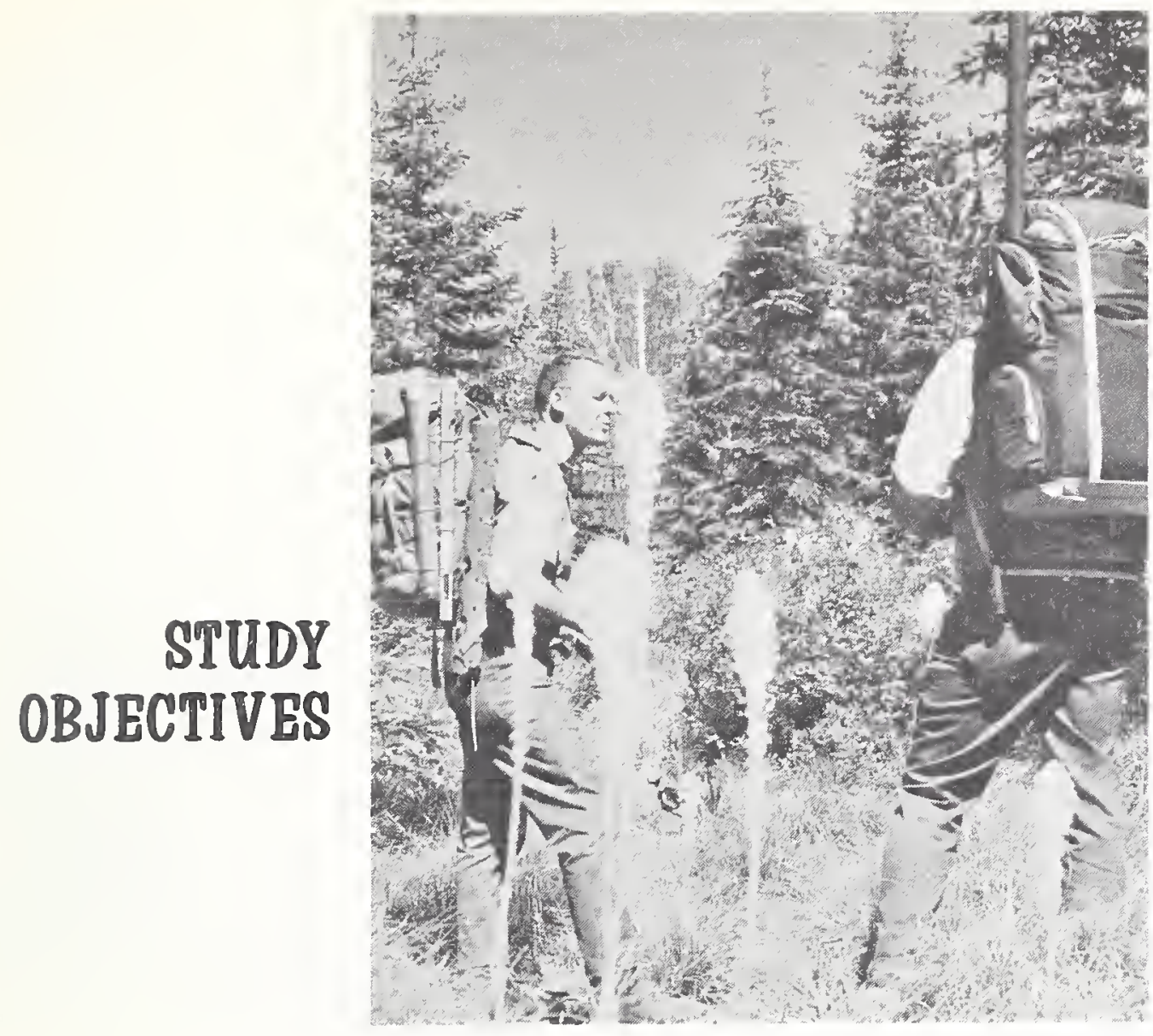

How can trail

register data best be converted into estimates of wilderness use?

The most accurate information on wilderness use would probably be obtained from use of a registration or permit system administered directly by the area managers. The California Region of the Forest Service began requiring registration for wilderness visitors in 1971. This is costly; for most wildernesses, an unmanned self-registration system seems more feasible for now. But everyone does not register. As a result, registration data cannot be taken at face value; they must be converted to estimates of total use. How to make this conversion is the central objective of this study. This required (1) developing and testing a sampling design to provide estimates of current use, and (2) establishing relationships between use and trail register data so that use estimates with specifiable levels of precision could be made in future years, based on register data.

Several sorts of automatic counting devices were also tested. To aid in developing use estimation methods applicable to other wilderness-type areas having different kinds of use and varying conditions, we investigated the relationship of registration rates to visitor characteristics and to other factors such as season and weather. To help raise registration rates, we probed reasons for nonregistration.

The Mission Mountains Primitive Area was selected for study because it was large enough and complex enough to offer a thorough test of the sampling model but not so large as to make fieldwork for a pilot test too formidable. The Area's administrators felt it had a substantial amount of both foot and horse travel, day and overnight use, and summer and fall use; thus, the area seemed to be representative in important ways of many wilderness-type areas within the National Forest System. 


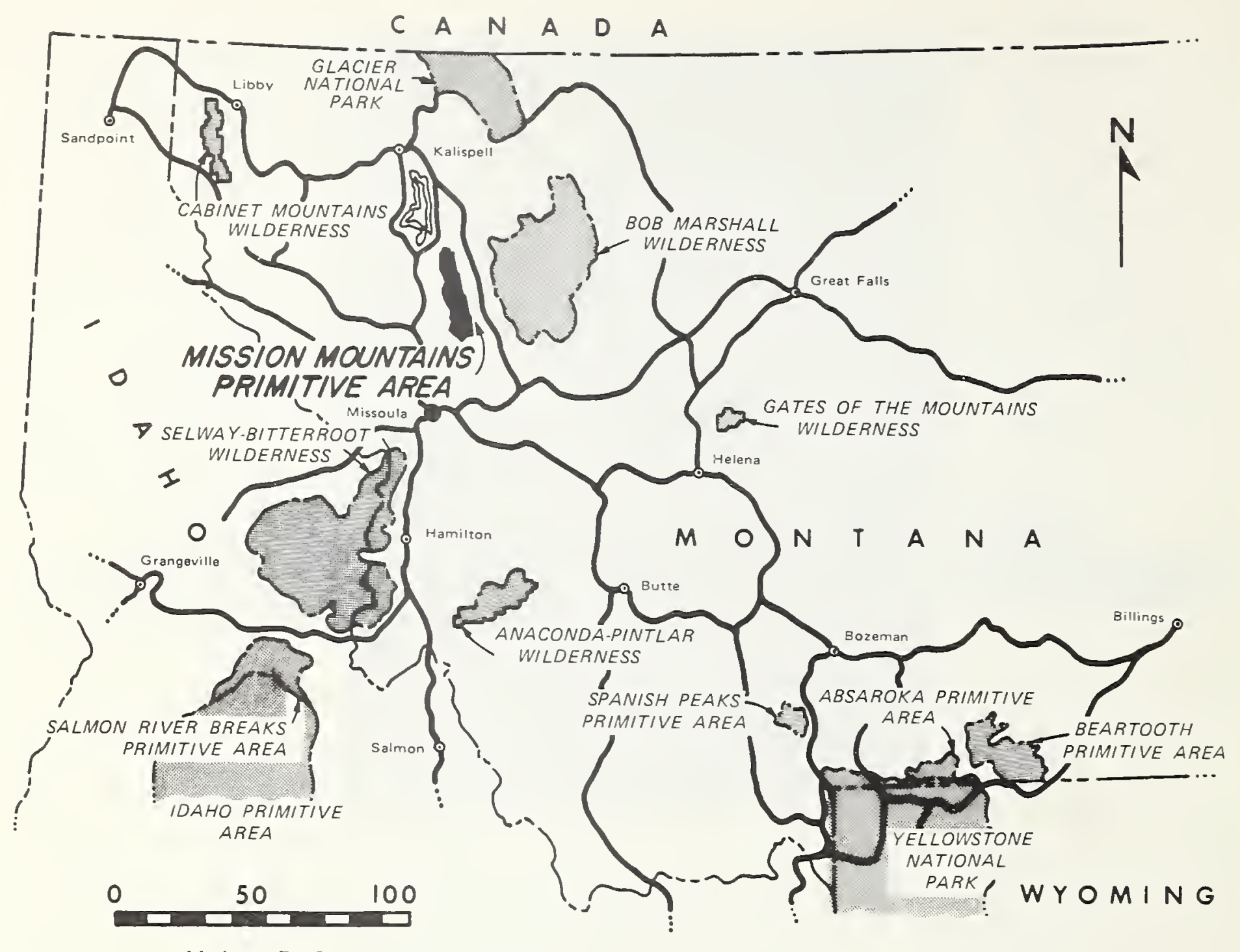

$M I L E S$

Figure 1. - Map showing location of study area in the Northern Rockies.

\section{The Study Area}

The Mission Mountains Primitive Area is situated on the east slope of the Mission Mountain Range encompassing approximately 75,000 acres (fig. 1). The high country west of the crest, equally wild and beautiful, is in the Flathead Indian Reservation. The Mission Range is narrow and high. Peaks rise up to 10,000 feet and tower more than 6,000 feet above the Swan Valley to the east and the Mission Valley to the west. It is a very rugged range, having steep, deep, and narrow canyons. Small glaciers lie on several peaks. Mountain goats, grizzly and black bear, deer, elk, coyotes, mountain lions, and grouse are among the varied wildlife. Over 130 lakes provide a total of about 2,400 acres of water surface (fig. 2). Some lakes are barren; others offer good fishing. Streams generally are very rapid, often brush-choked, and usually provide poor fishing (fig. 3). The trail system is rather limited. Most of the trails are dead-end routes up the deep, east-west canyons to the high cirque lakes. North-south trails, loop trails, or trail connections from one drainage to the next are scarce. About half of the trails within the Primitive Area and almost all on the west side of the Mission Mountain Range are old Indian or packer trails, usually rough, steep, and unsigned. Campsites are not abundant, and horse feed is very scarce (fig. 4). There are about 20 access points, including six trails through passes from the Indian Reservation to the west. 


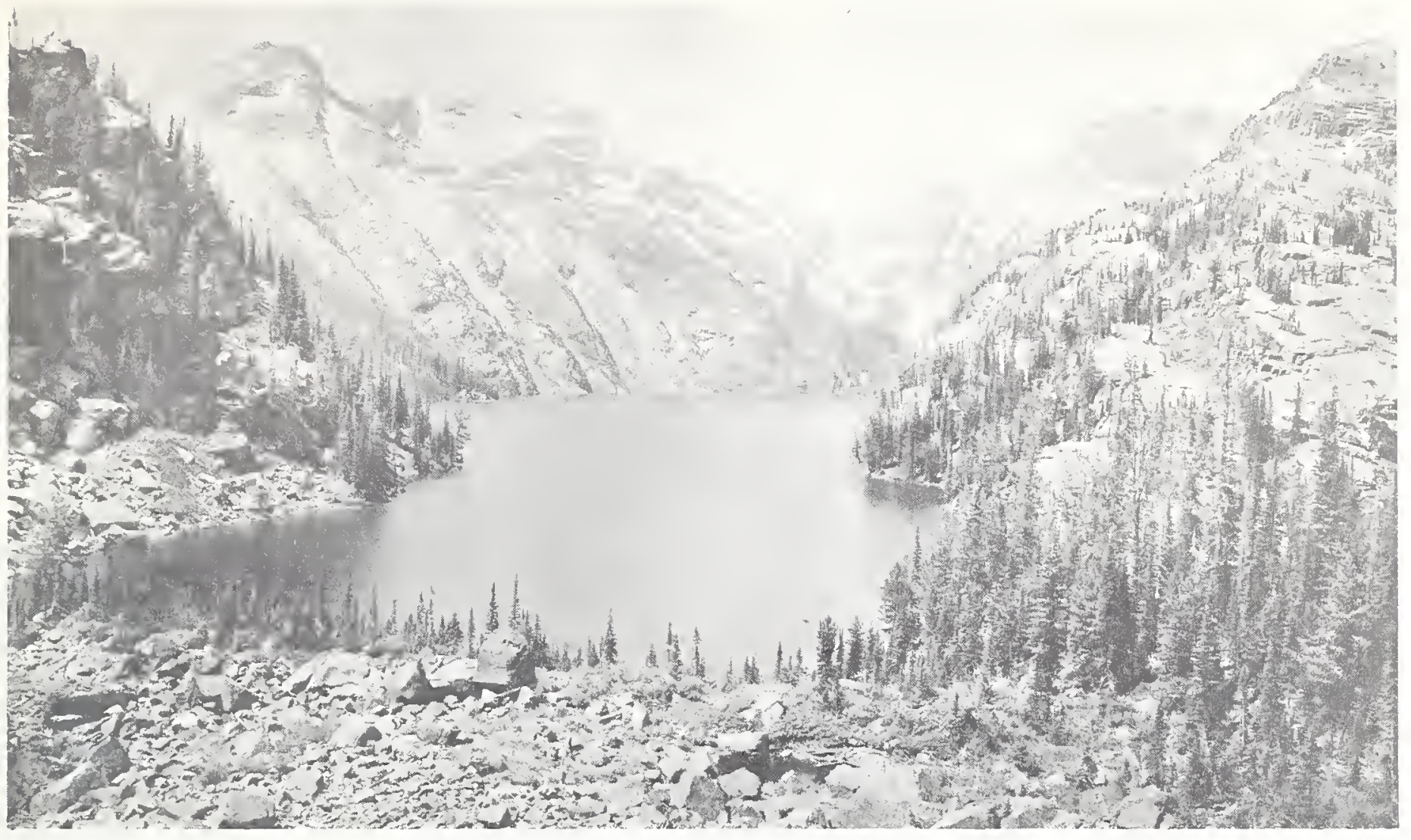

Figure 2, - The Mission Range is narrow, but high and rugged. There are a few small glaciers and over 130 lakes. Turquoise Lake and Sunrise Glacier are shown here.

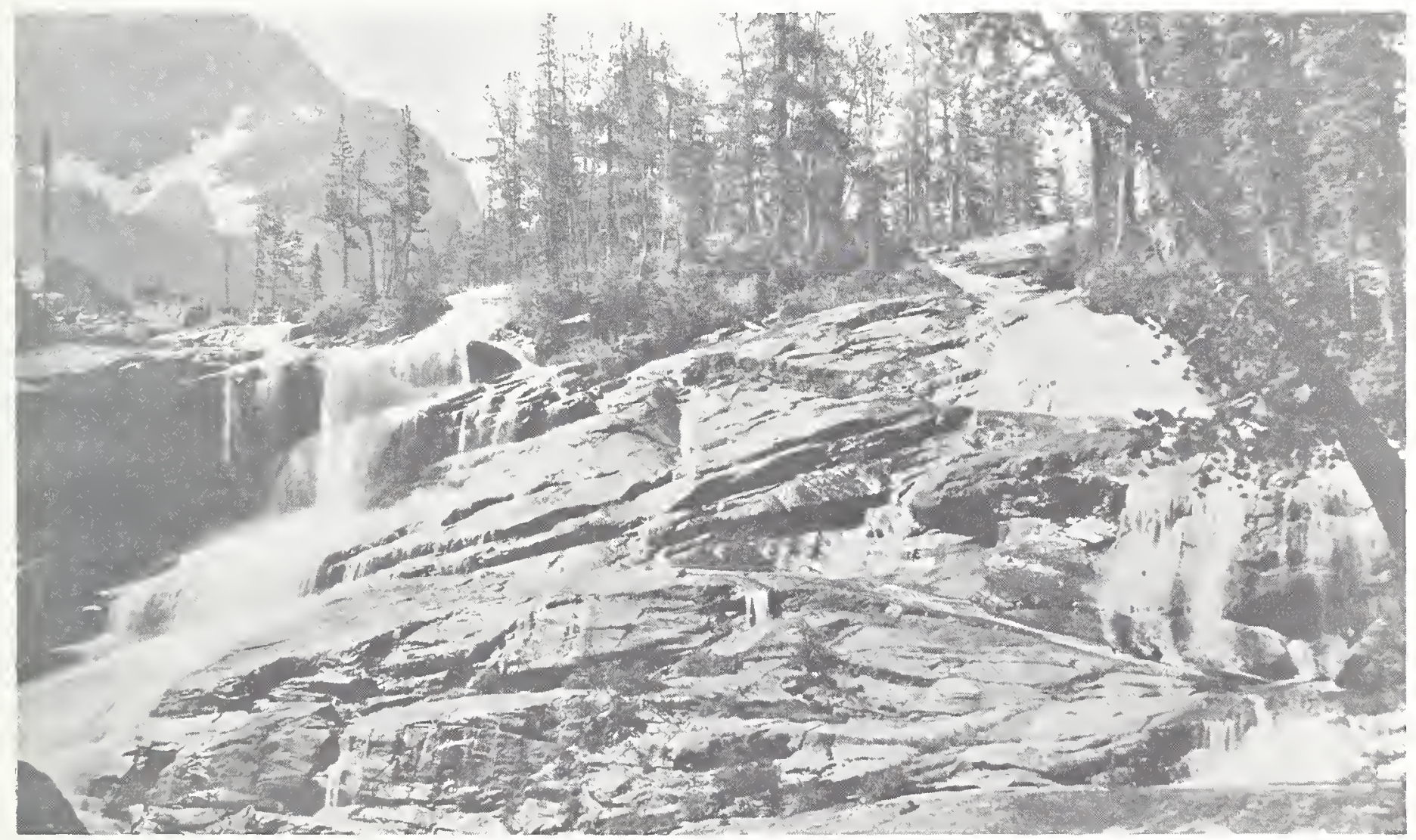

Figure 3. - The streams in the Mission Mountains are often beautiful cascades, such as this one above Glacier Lake, but they are usually too rapid and brush-choked to provide good fishing. 


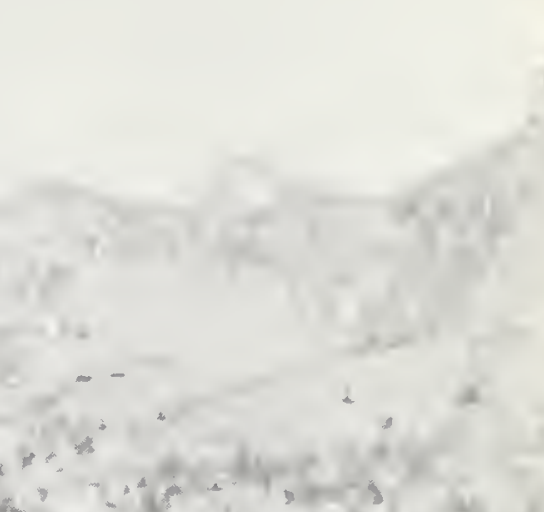

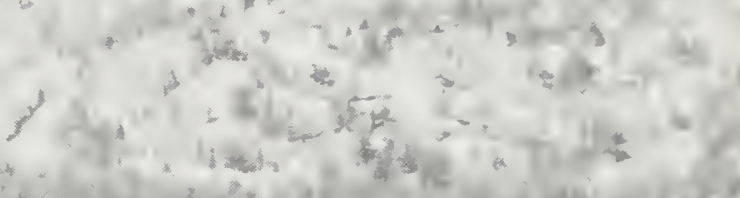

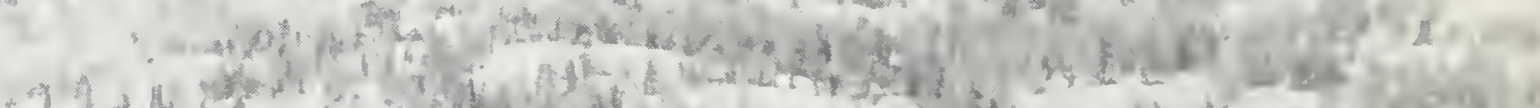

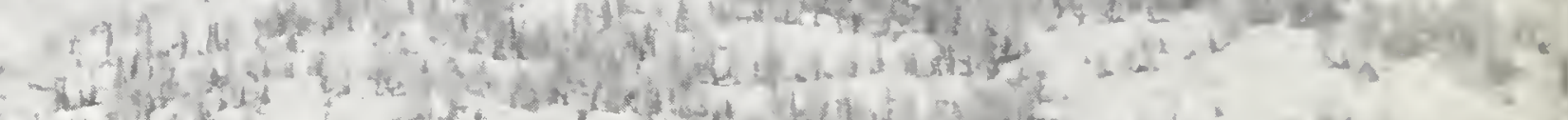

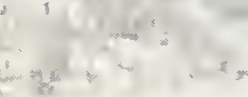

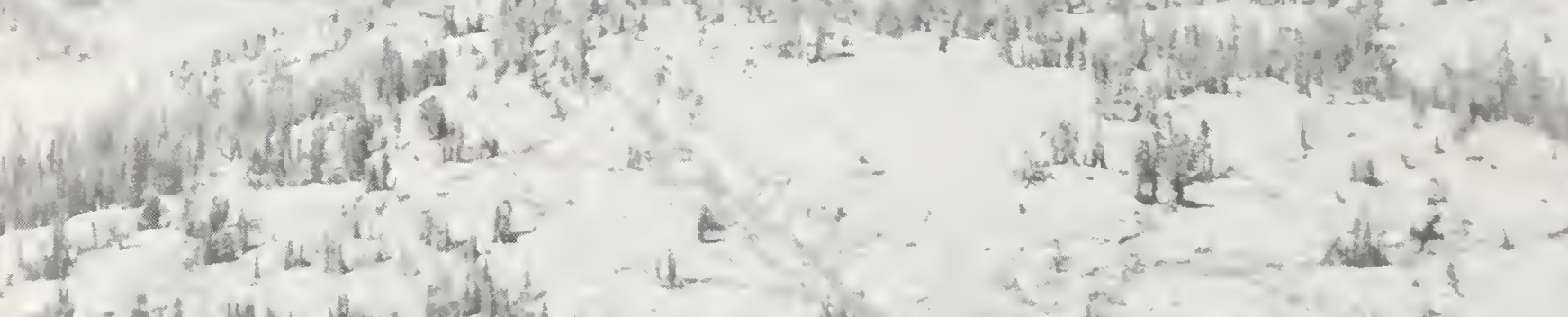

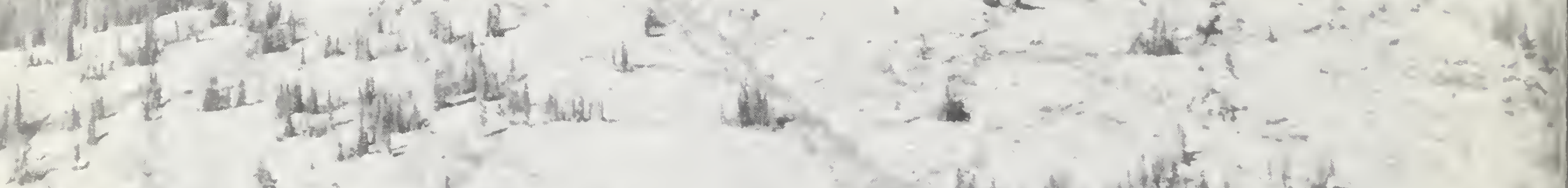

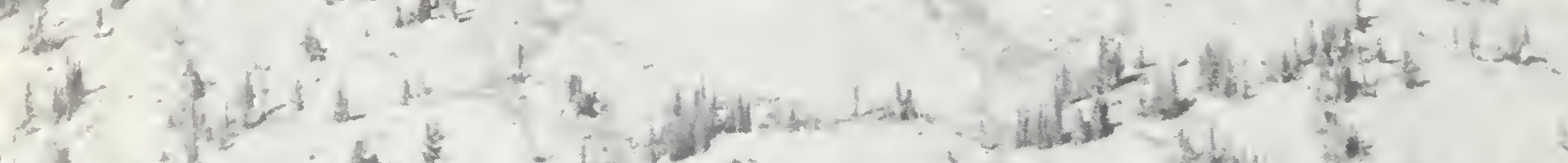

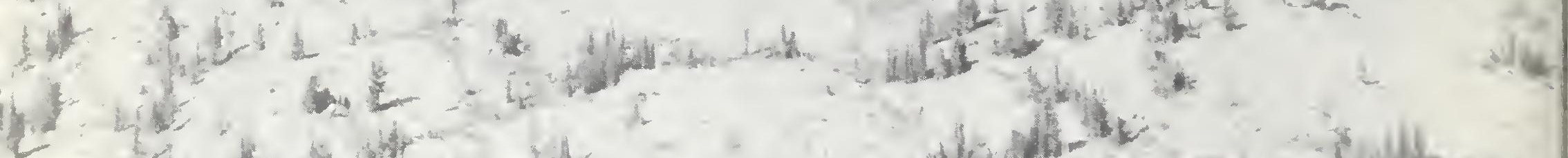

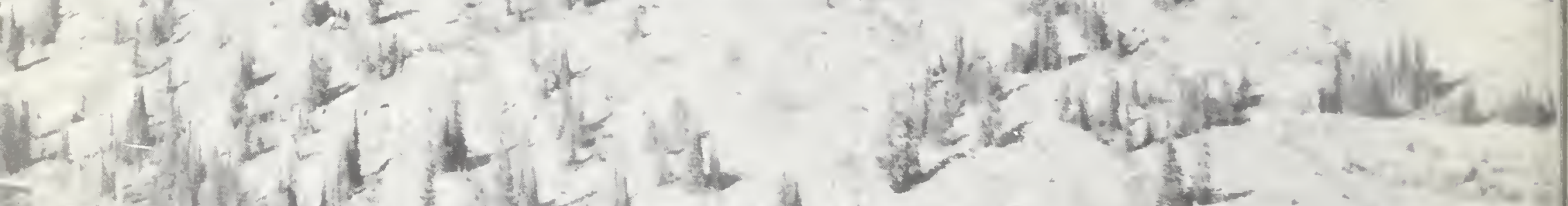
- $110 x^{2}+h^{2}$ 2.

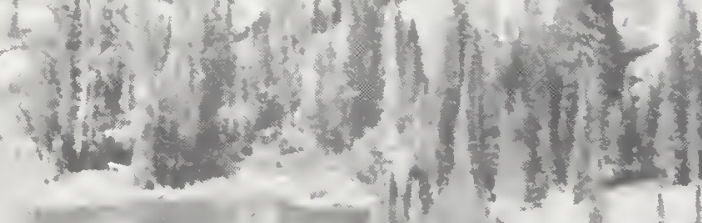
7) Nule

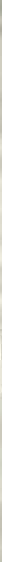




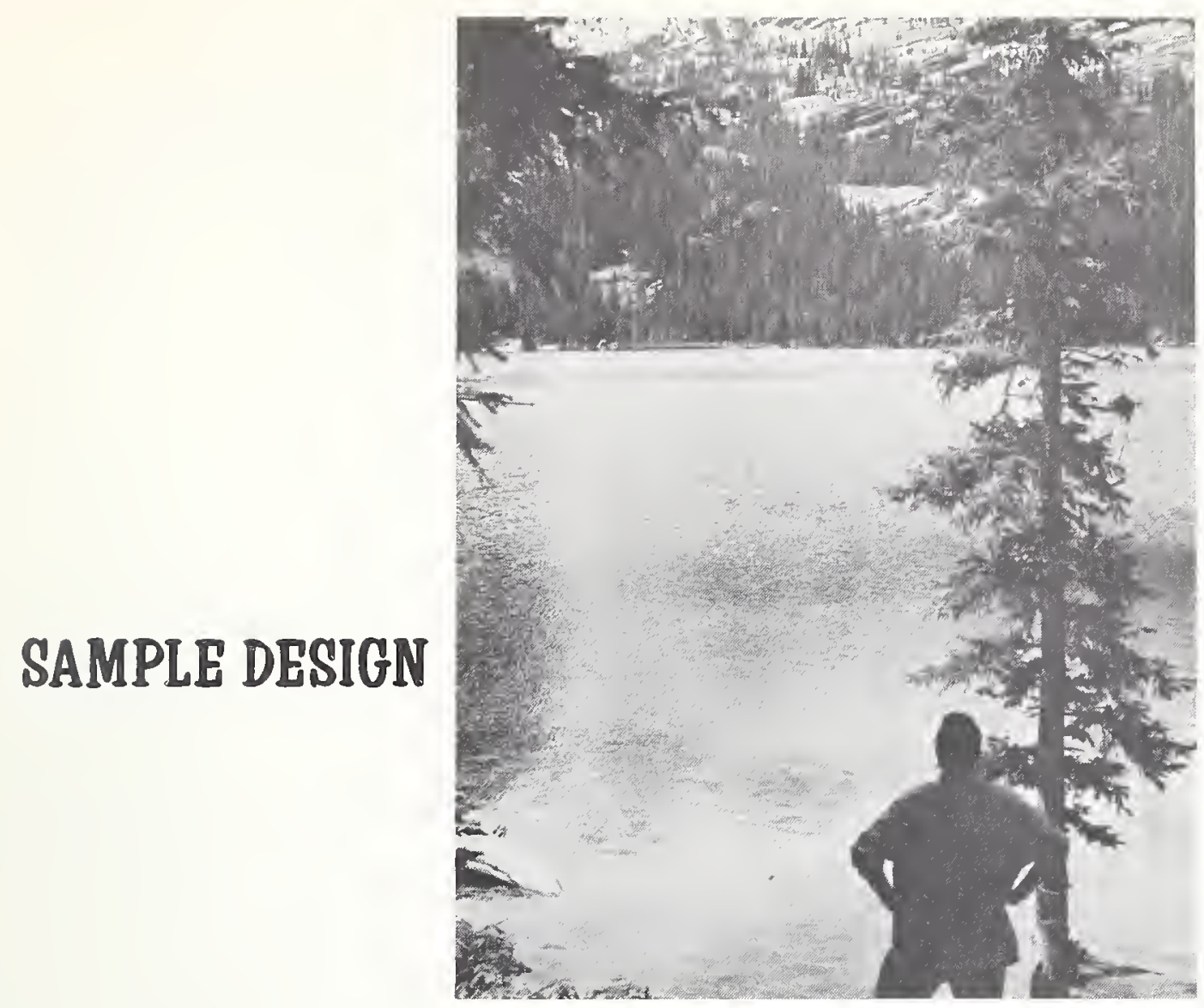

Sampling was concentrated on more heavily used trails. The trail to Glacier Lake, shown here, was by far the most popular. It accounted for close to half of all group visits. It was also the most heavily sampled trail.

The basic sample design was stratified sampling. This concentrated field time on trails having enough use to enable us to observe the registration behavior of visitors; thus we reduced the number of wasted sample days that didn't yield any data. Trail use was expected to have a highly skewed distribution--a few trails heavily used and many very lightly used. We also suspected that some types of trails might have different registration patterns at certain times.

Seven strata were established, differentiated on the basis of varying combinations of three variables: season (summer or fall), time of week (weekday or weekend), and expected use on trails (see table 1). Trails were placed in one of four classes of use on the basis of judgment estimates made by Forest Service Ranger District personnel. The four classes were $1 / 4$ to 1 (very light), 1 to 2 (light), 2 to 5 (medium), or over 5 (heavy) visits per day, on the average. Trails estimated to average less than onefourth of a visit per day were not included (table 1).

Two-day sampling units, rather than l-day units, were used to reduce (a) the frequency of zero observations, (b) travel expense, and (c) variability in the estimation equations. The number of sample units allocated to each strata was based on the following: (a) The expectation that heavy-use strata would be more variable than lightuse strata; (b) that interviewing costs would be much less in the heavy-use strata due to more visitor contacts per workday; and (c) that use estimates were desired for individual trails. We felt that 11 to 18 sampling units in each stratum would produce an adequate regression estimate for each trail even if the corresponding estimates of precision (the estimates of error terms) might be unreliable. Lightly used trails were intentionally sampled at a lower sampling intensity than believed desirable for statistical reasons because more intensive sampling would not have been economically feasible. The numbers of trails and 2-day sample units in each strata are shown in table 1. 
Table 1.--Definition of strata and somple selection

\begin{tabular}{|c|c|c|c|c|c|c|}
\hline \multirow{2}{*}{$\begin{array}{r}\text { Stratum } \\
\text { number }\end{array}$} & \multicolumn{4}{|c|}{ Combination of variables } & \multicolumn{2}{|c|}{ Allocation of sampling } \\
\hline & Season ${ }^{1}$ & : Time of week & : Estimated use & : Trails & $\begin{array}{c}\text { : Total sample } \\
\text { units }\end{array}$ & $\begin{array}{c}\text { Sample units } \\
\text { selected }\end{array}$ \\
\hline & & & Persons per day & Number & Inumber & Inumber \\
\hline 1 & Summer & $\begin{array}{l}\text { Weekend/ } \\
\text { holiday }\end{array}$ & $>5$ & 1 & 14 & $12(86)$ \\
\hline 2 & Summer & Weekdays & $>5$ & 1 & 26 & $12(46)$ \\
\hline 3 & Summer & All days & $2-5$ & 1 & 40 & $14(35)$ \\
\hline 4 & Summer & All days & $1 / 4-2$ & 16 & 340 & $18(5)$ \\
\hline 5 & Fall & $\begin{array}{l}\text { Weekend/ } \\
\text { holiday }\end{array}$ & $2-5$ & 5 & 45 & $13(29)$ \\
\hline 6 & Fall & Weekdays & $1-5$ & 9 & 207 & $30(15)$ \\
\hline 7 & Fall & $\begin{array}{l}\text { Weekend/ } \\
\text { holiday }\end{array}$ & $1 / 4-2$ & 13 & 117 & $11(9)$ \\
\hline
\end{tabular}

ISummer season was from June 15 to September 2, inclusive. Fall season was from September 9 to November 11, inclusive. In general, the end of the season is determined by the onset of winter so that the November 11 date was known only after the season actually closed. The hunting season for mountain goats opened on september 15, and about 100 permits were issued. Most of the area opened for either sex elk hunting beginning September 21; the extreme northern part opened on October 27. Deer hunting opened October 27. Fishing was permitted during the entire study period.

2 Sampling intensity in percent is shown in parentheses.

\section{Sample Selection}

Sample units were randomly selected within the constraints of feasible work scheduling. On weekdays, one location was sampled. On weekends and holidays, two locations were sampled. We selected 110 two-day sampling units (table 1): 56 units during the sumner and 54 in the fall. First, dates were chosen randomly for each stratum, and then a trail location was randomly chosen for each date. Sample dates were chosen first for stratum 1, then for stratum 2, and so on from the remaining available workdays. 


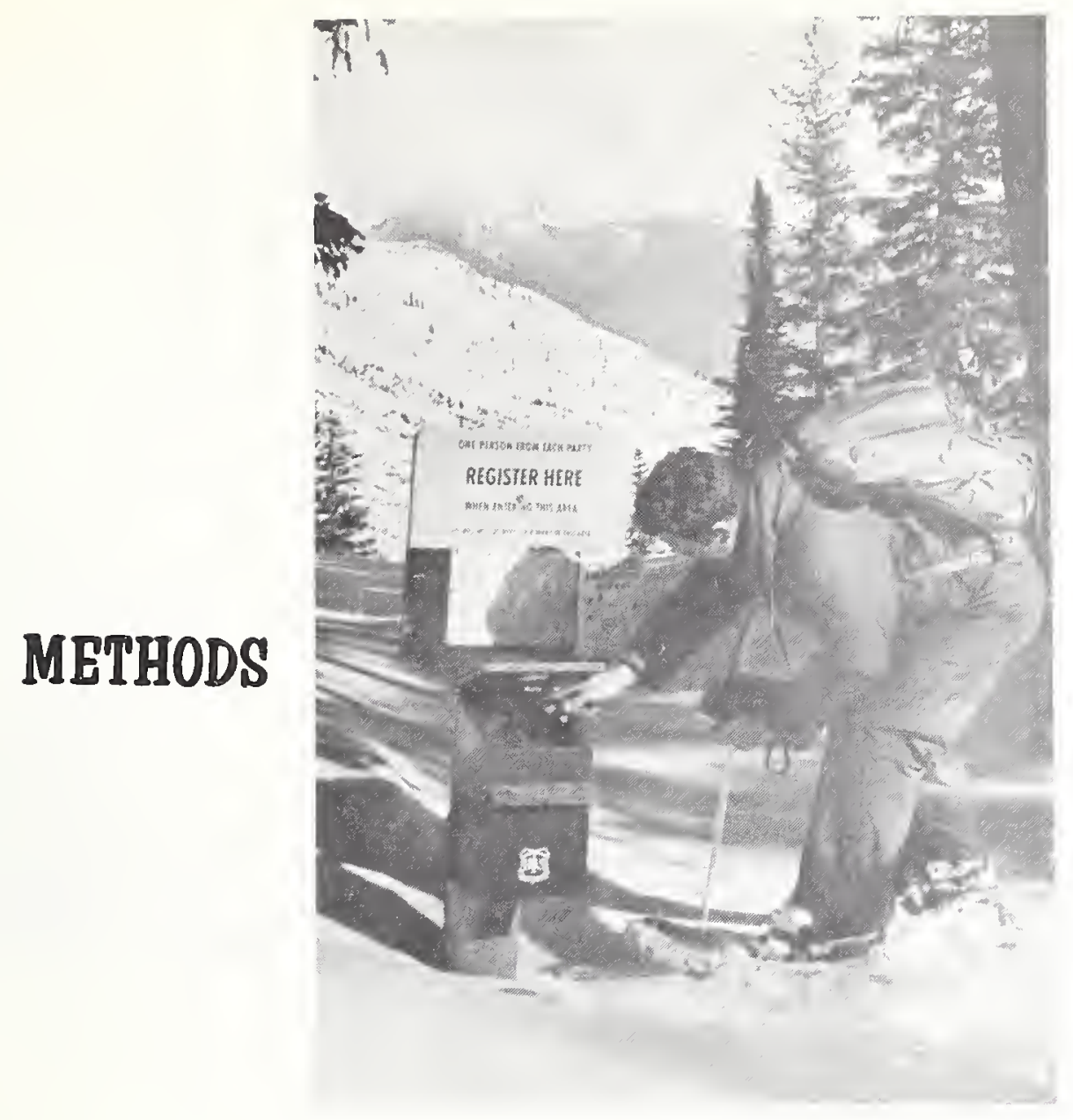

An 18-mile round trip, much of it on snowshoes, was required to service this self-registration station at Elk Pass in late October.

The variables in each stratum were measured using a questionnaire for interviews with groups entering or leaving (fig. 5). Supplementary (covariate) information was obtained by establishing registration boxes on each trail, where entering groups could register by filling out a wilderness registration card (Appendix I). This was a condensed version of the questionnaire used for trail interviews. Mechanical counters placed on some of the most heavily used trails and access roads provided additional covariate data.

\section{Interviewing Procedures}

Four interviewers worked as two-man teams checking sample locations from 8 a.m. to 8 p.m. from June 15 until October 20. From October 20 through November 11 the checking period was shortened to 12 noon to $6 \mathrm{p} . \mathrm{m}$. because the reduction in daylight hours coupled with changes in use lessened the chances for missing parties. This made it possible to check twice as many locations each day during the last 3 weeks--four locations on weekends, two on weekdays.

Interviewers stationed themselves up the trail, out of sight of the registration station. They drove personal cars rather than official Forest Service vehicles to avoid influencing visitor registration. Interviewers were dressed in official uniform. They conducted a brief interview with every party that entered or left. 


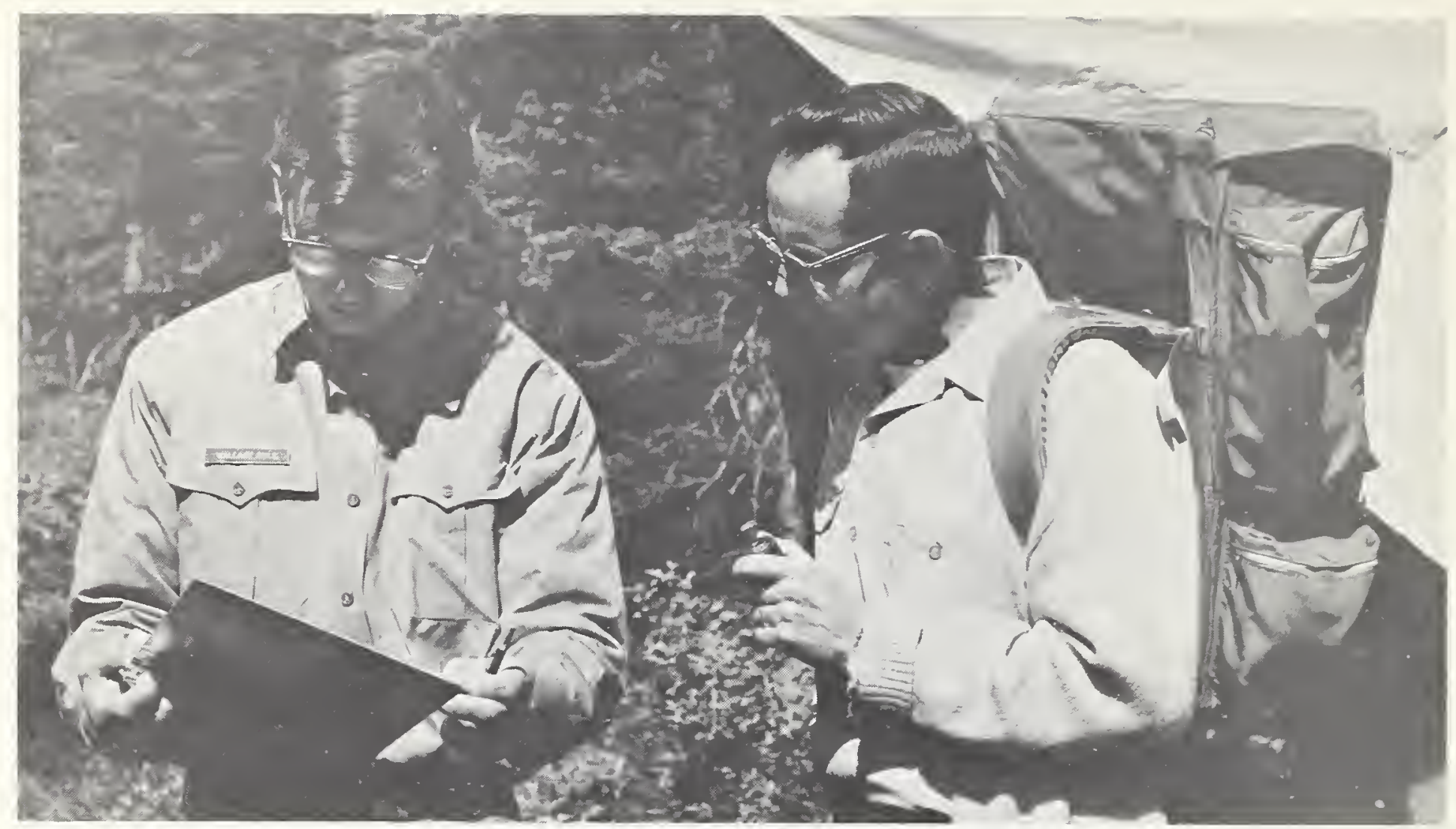

Figure 5. - Brief interviews were conducted with every party entering or leaving during sample periods to establish actual use to compare and relate to registered use. Sampling was intensive; close to half of the 1968 visitors were contacted.

\section{Registration Stations}

Unmanned registration stations (fig. 6) were installed at 19 trail entrances (fig. 7) in early June, except for trails through the passes; snow made these inaccessible until late June and early July. The locations for these stations were selected in consultation with the Ranger District staff and included all trails thought to be used by at least an average of one group every 2 weeks (roughly equal to one-fourth of a visit per day). One additional trail meeting this criterion was discovered and added several weeks after the study began; it leads from the Indian Reservation south of the Primitive Area to Gray Wolf Lake. The registration stations were located where the trails began at the road, rather than on the Primitive Area boundary (except for trails through the passes).

Vandalism of unmanned registration boxes as well as of signs, counters, and other equipment was not a problem. However, two registration stations were severely damaged by porcupines. Several more stations suffered similar damage, although it was minor. After stovepipe was placed around posts supporting the signs and boxes, porcupines gave us no more trouble.

The registration box, sign, map on signboard, and card format that we used all were based on Wenger (1964).2 The sign directed one person from each group to register for the entire party when entering. The registration card (Appendix I) asked for a

${ }^{2}$ The sign, however, following current Forest Service standards, was gray and brown instead of yellow and black as in Wenger's test. 


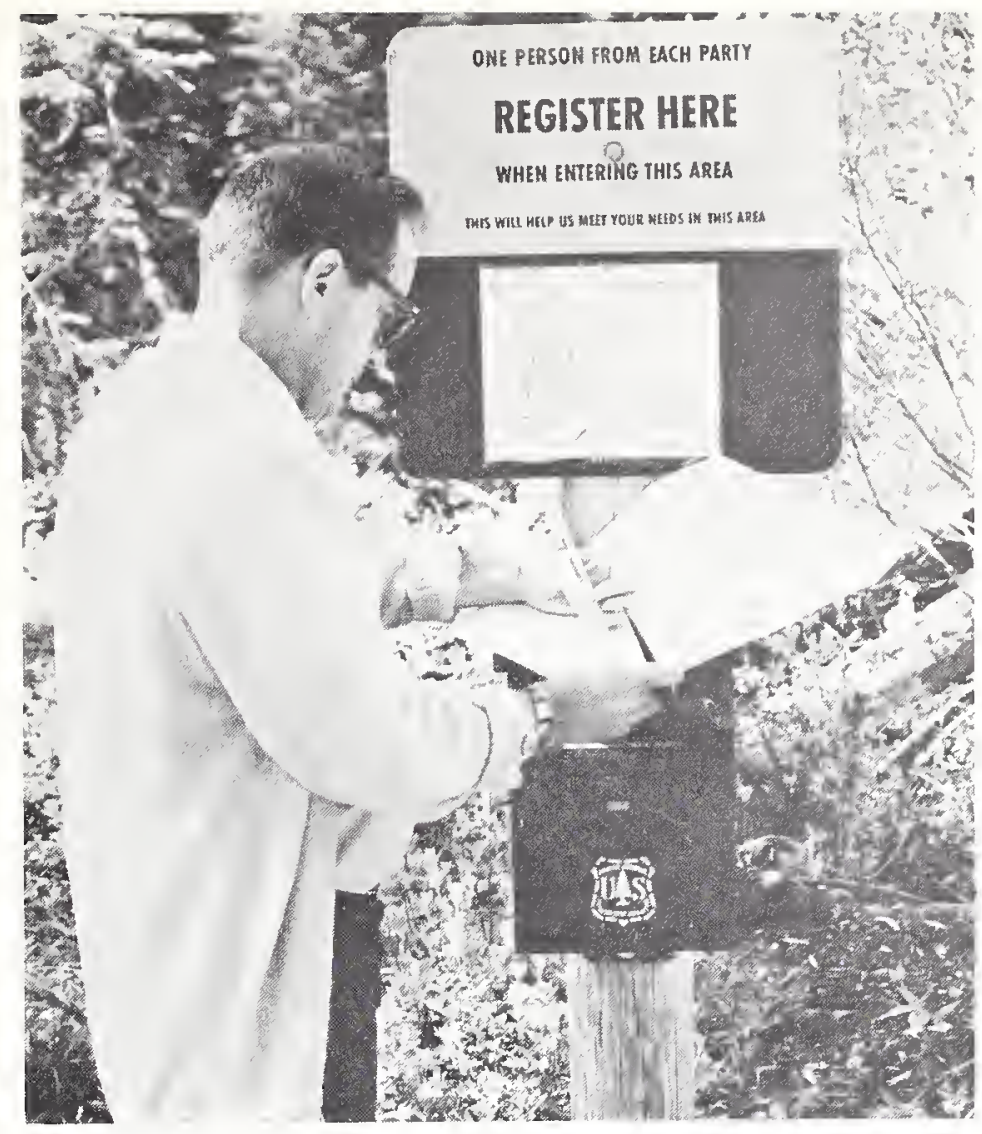

Figure 6. - An estimated 65 percent of all groups registered in the Mission Mountains Primitive Area.

standard trip description: name, address, party size, date and time in, planned date and time out, number of pack and saddle animals, whether an outfitter or guide was used, number of people planning certain activities, the numbers of the "compartments" in which they planned to travel, and locations where they planned to camp if they were staying overnight. The registration card was accompanied by a folded map showing 27 numbered "travel compartments." The compartments were based on trail patterns, usually conforming to drainage basins. The lower and upper halves of drainages were generally divided into separate compartments.

Registration cards were collected on each sampling day and stapled to corresponding interview forms for punching. Registration boxes were serviced at least once each week, except those in the mountain passes, which were serviced either two or three times during the study.

\section{Traffic Counters}

Conventional nonprinting pneumatic traffic counters were installed on the access roads to the two most used trails: Glacier Lake (number 1 on fig. 7), and Cold Lake (number 2 on fig. 7). A pressure-pad adaptation of a similar traffic counter was installed on the Glacier Lake trail. Four prototype ultrasonic beam trail traffic counters were installed: two on the Glacier Lake trail; one on the Cold Lake Trail; and one on Fatty Creek trail (number 3 on fig. 7), which was the next most heavily used trail. Counters were read three times each day when the location was sampled, and every third or fourth day at other times. 


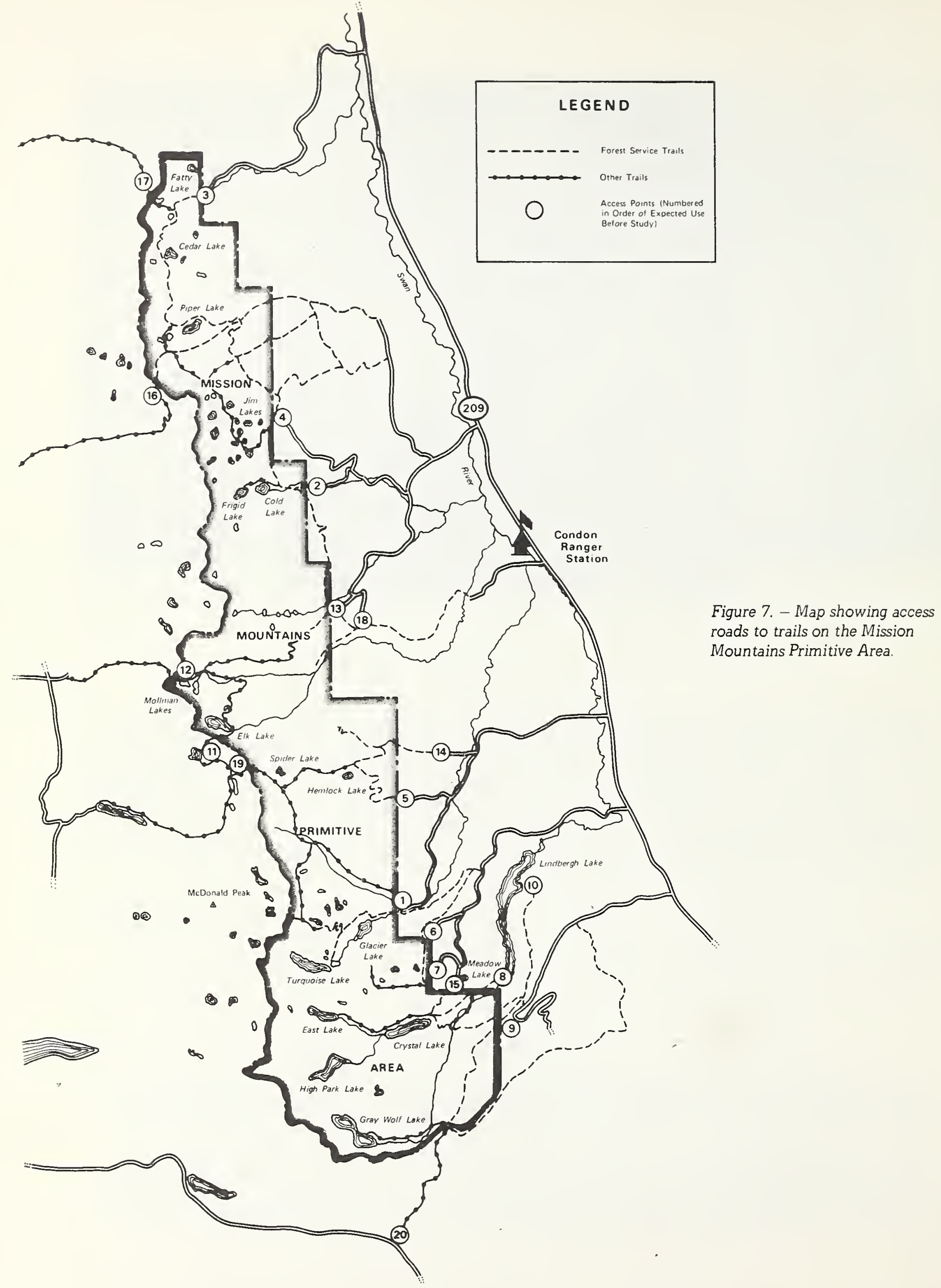




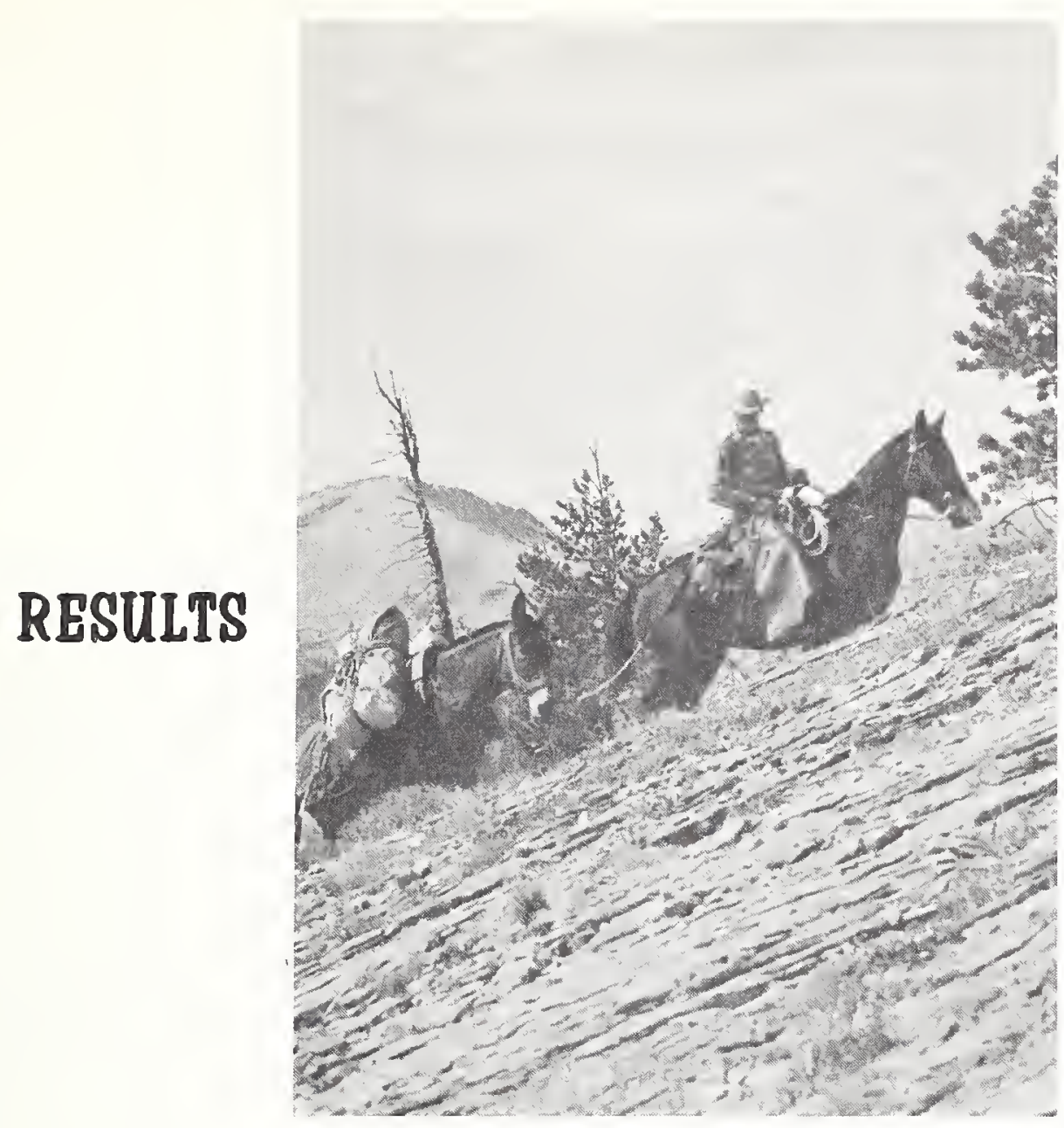

Only about 5 percent of the visitors traveled by horse, largely due to rough terrain and the scarcity of forage. Even fewer parties traveled with guides or outfitters.

Visitors responded well to the personal interview situation. There seemed to be no resentment or problem with visitors feeling "spied upon" concerning registration. No one refused to answer questions.

In total, 470 groups were interviewed, 312 of which were interviewed both when entering and when leaving. This produced a total of 782 interviews (table 2).

\section{Registration Rates}

A key question asked all interviewed groups was: "Did your party register upon entering the area?" Registration forms were checked to see if the answers to this question were truthful. In more than 95 percent of the cases, groups who had not registered admitted honestly that they had not.

An estimated 65 percent of all groups registered. This registration rate is fairly close to the 74-percent registration rate for the two wildernesses studied by Wenger and Gregersen (1964). Although the Mission Mountains Primitive Area is more than 500 miles from the area studied by Wenger and Gregersen and does not draw visitors from the same geographical region, both are predominantly day-use hiking areas that serve visitors largely from nearby regions rather than from the entire nation. 
Table 2.-Nomber of groups interviewed by stratum

\begin{tabular}{|c|c|c|c|c|}
\hline $\begin{array}{l}\text { Stratum } \\
\text { number }\end{array}$ & $\begin{array}{l}: \text { Entering } \\
: \quad \text { only } \\
\end{array}$ & $\begin{array}{l}\text { : Leaving } \\
: \text { only }\end{array}$ & $\begin{array}{l}: \text { Both entering } \\
: \text { and leaving }\end{array}$ & $\begin{array}{l}: \text { At least } \\
: \text { once }\end{array}$ \\
\hline 1 & 21 & 39 & 142 & 202 \\
\hline 2 & 16 & 16 & 81 & 113 \\
\hline 3 & 9 & 26 & 47 & 82 \\
\hline 4 & 3 & 2 & 7 & 12 \\
\hline 5 & 3 & 3 & 25 & 31 \\
\hline 6 & 4 & 10 & 9 & 23 \\
\hline 7 & 2 & 4 & 1 & 7 \\
\hline Totals & 58 & 100 & 312 & 470 \\
\hline
\end{tabular}

The registration rate was depressed by stratum 7 (fall weekends on lightly and moderately used trails) where only seven groups were contacted, none of whom had registered. Registration rates, by stratum, were:

$\begin{array}{cr}\begin{array}{c}\text { Estimated } \\ \text { registration rate }\end{array} & \text { Standard } \\ \text { (Percent) } & \text { error }{ }^{3}\end{array}$

\begin{tabular}{|c|c|c|}
\hline SUM & & \\
\hline$S t$ & & \\
\hline 1 & 69 & 2 \\
\hline 2 & 76 & 4 \\
\hline 3 & 68 & 6 \\
\hline 4 & 83 & 20 \\
\hline
\end{tabular}

FALL

Stratum 5

6

7

$\begin{array}{rr}58 & 9 \\ 57 & 10 \\ 0 & 0\end{array}$

Summer strata pooled ${ }^{3} \quad 74 \quad 7$

Fall strata pooled ${ }^{3} \quad 41 \quad 19$

All strata pooled ${ }^{3} \quad 65 \quad 6$

Use was even more heavily concentrated on a few trails than had been expected; as a result of this and the greater sampling intensity for heavy-use strata, 93 percent of all interviews occurred on two trails. Only five trails yielded five or

${ }^{3} \mathrm{~A}$ confidence interval of plus or minus one standard error around the estimated mean will contain the true mean about two-thirds of the time. Plus or minus 2 standard errors will contain the true mean about 95 percent of the time. See Appendix II for the formula (from Cochran, 1963, section 6.11) that was used to calculate standard errors. 
more interviews. The registration rate and number of groups interviewed at least once are shown in the following tabulation for all trails on which at least one interview was obtained (see fig. 7 for trail locations).

\section{Trail nome and number}

$\begin{array}{lr}\text { Glacier Lake } & 1 \\ \text { Cold Lakes } & 2 \\ \text { Fatty Creek } & 3 \\ \text { Jim Lakes } & 4 \\ \text { Red Butte } & 5 \\ \text { Skylark Lake } & 7 \\ \text { S. Lindbergh Lake } & 8 \\ \text { Beaver Creek } & 9 \\ \text { Mollman Pass } & 12 \\ \text { S. Fork Cold Creek } & 13 \\ \text { Hemlock Creek } & 14 \\ \text { Meadow Lake } & 15 \\ \text { Elk Creek } & 18\end{array}$

\section{$\frac{\text { Registration rate }}{(\text { Percent })^{4}}$}

71

67

73

22

100

0

50

87

100

33

0

100
Number interviewed

346
89
5
4
2
1
4
5
1
6
2
1
4

\section{Completeness of Response on Registration Cards}

The key items on the registration card were completed by a high percentage of the registrants as shown in the following tabulation:

Item

Name and address

(sufficient to address mail)

ZIP code

Date entered area

Time entered area

Date left area (planned)

Time left area (planned)

Number of persons in group

Number of saddle animals

Number of pack animals

Accompanied by guide ("yes" or "no" checked)

Travel compartment numbers (planned)

Campsite locations (planned)

Activities (planned)
Items

completed

(Percent)

\footnotetext{
${ }^{4}$ These are weighted means wherever the sample groups came from more than one stratum; thus four groups were contacted at Jim Lakes and the estimated registration rate is 22 percent, rather than 25 , because of the weighting of the strata involved. ${ }^{5}$ Percentage of parties traveling with stock who indicated the number of animals. ${ }^{6}$ Tabulations are based only on those visitors staying overnight in the area.

${ }^{7}$ This question was apparently misunderstood. Instead of indicating the number of persons in each group who planned to participate in each of the activities listed, 73 percent indicated the activities that someone in the group planned to pursue. Seventeen percent did not answer.
} 


\section{Accuracy of Planned Length of Stay}

Signs at registration stations directed visitors to register when entering. This meant that visitors could only indicate expected rather than actual time of departure. Many factors, such as adverse weather and unexpected trail conditions, upset visitors' time schedules. On the average, visitors who registered did not stay quite as long as they had expected. Visitors who did not register, but who were interviewed both upon entering and leaving, overestimated their stay by approximately 5 percent. About 61 percent of all registered groups who were interviewed upon exiting estimated the length of their visit correctly (when both the planned and actual stay were expressed to the nearest whole hour). Groups that came out earlier than planned made up 23 percent of the total, and parties that stayed longer than planned accounted for 16 percent. Generally, short stays (under 5 hours) were longer than expected, while longer visits tended to be shorter than planned. Expected length of stay exceeded actual length of stay by 10 percent for those registered groups who were interviewed upon exiting.

\section{Relationships of Visitor Characteristics, Other Variables to Rates}

A11 groups do not register; furthermore, certain types of visitors are less likely to register than others. Therefore, the raw registration data not only gives an incomplete picture of the use of an area but also a distorted view. Information such as the following on how a number of factors were related to registration rates could aid managers in interpreting registration data and also in planning ways to raise registration rates, which is an important management objective.

Sex and age.--Groups containing adult females generally registered at higher rates than did all-male or teenage groups (table 3 ). The groups that were made up entirely of adult males had an estimated registration rate ( 66 percent) about equal to the overall average. All of the types of groups containing teenage boys or girls had belowaverage rates (with the one exception of mixed adult and teenage females, in which case only two groups were interviewed and both had registered).

Size of group.--The medium-sized groups (3 to 5 members) had higher estimated registration rates than either the small or large groups (table 3 ). One-man groups (all one-person groups were male) had the lowest registration rate of all, but those who did register furnished more complete information than did larger groups. All oneperson registrations contained complete addresses, 84 percent provided ZIP codes, and almost all gave date and time both in and out, travel compartment numbers, and planned campsites. Presumably, the lone individual who registers sees some possible security value in providing full information about his wilderness travel plans.

Length of stay.--Visitors who stayed overnight registered at a higher rate than visitors who did not (table 3 ). The shorter the stay, the less likely were visitors to register.

Place of residence.--Local residents (only 8 percent of all interviewed groups) had a slightly lower registration rate than visitors who lived outside the local area (table 3). There was no discernible difference in registration rates between Montana residents and out-of-State visitors.

Weather conditions.--Interviewers subjectively classified the weather conditions at the time of the interview, and the registration rates for entering parties were related to the weather (table 3). Data from exiting parties were not used because weather conditions at the time they would have registered could no longer be observed. Temperature had little effect on registration rates. Rain and snow, however, reduced registration rates substantially. 


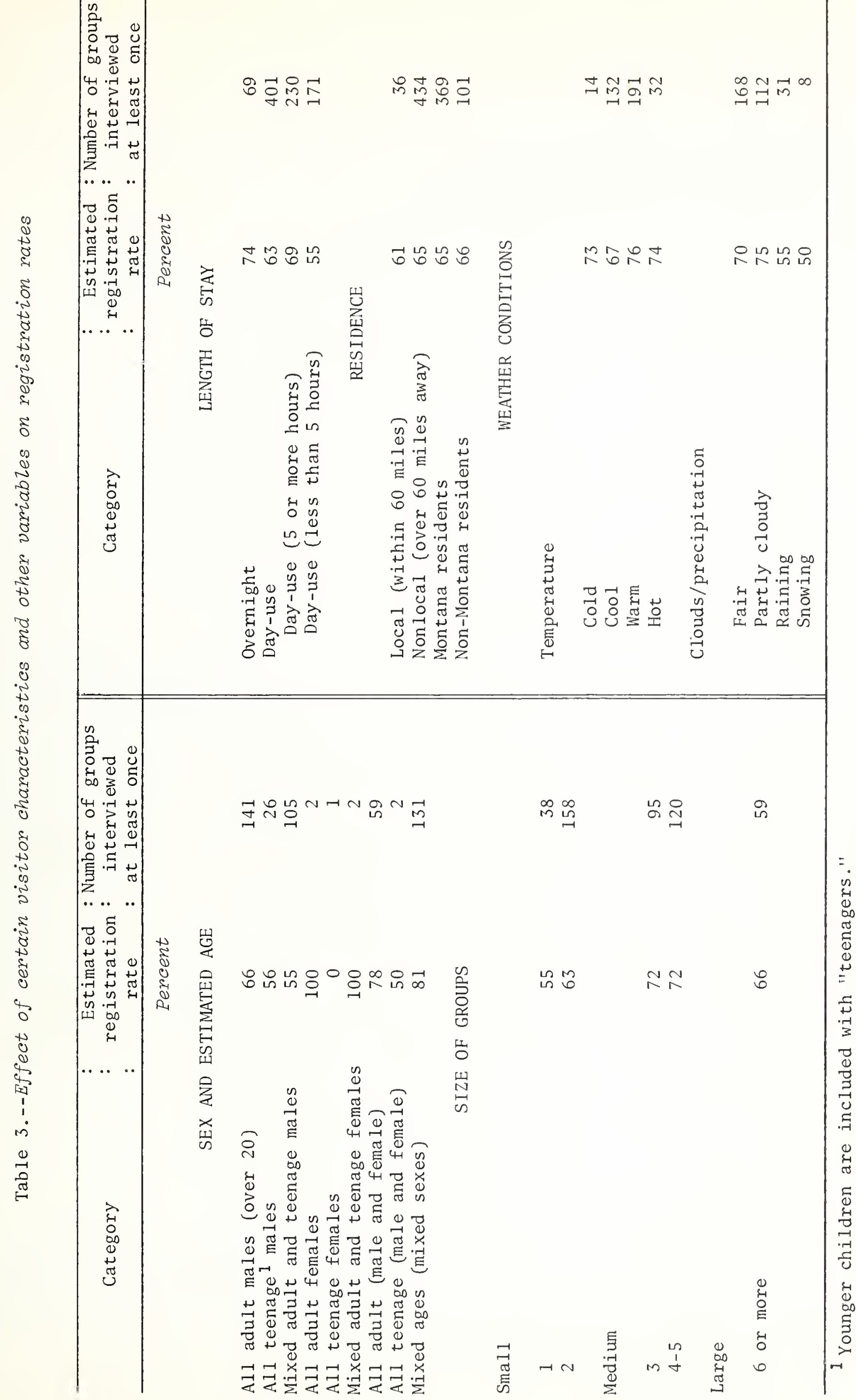


Kind of activity.--Groups that hunted had a low rate--only an estimated 34 percent registered (based on 38 hunter groups interviewed at least once). Groups having members who fished had a 75-percent rate (based on 357 fishing groups interviewed at least once). Groups that neither fished nor hunted had an intermediate rate of 54 percent (based on 75 groups interviewed).

Prior use during the season.--Groups were asked if most of the people in the group had visited the area during 1968, prior to the interview. More than half had visited the area earlier during the season. Familiarity with the area from previous visits lowered rates by only a small amount; the first-time visitor groups had an estimated rate of 69 percent (201 interviews), whereas groups that had made earlier visits had a 64 -percent rate (269 interviews).

Mode of travel.--Horsemen registered at a lower rate than hikers--an estimated 44 percent for horsemen compared to 66 percent for hikers. However, the horseman sample consisted of only 9 groups interviewed (against 461 hiker groups); thus, neither the horseman rate nor the difference between the two rates can be estimated precisely.

Use of outfitters or guides.--The sample of groups that used outfitters or guides included only 5 groups, only one of which registered. In contrast, an estimated 68 percent of the groups without outfitters or guides registered.

Time of week.--Weekday registration rates were estimated at 72 percent (157 interviews). Weekend and holiday rates were lower, 62 percent (313 interviews).

\section{Reasons for Not Registering}

About 32 percent $^{8}$ of the 470 groups who were interviewed did not register. These groups were asked by interviewers why they had not registered to try to learn if there is some way to raise registration rates. A tabulation of their replies follows:

$$
\text { Reasons for failing to register }
$$

In a hurry, did not want to take time

Planned to register on way out

Had registered on a previous visit, thought once per season was all that was required

Only going a short distance, did not think it necessary to register

Did not notice registration box and sign; in some cases because of darkness

Did not like to register

Said they had registered, but had not

Raining

Thought someone else in party had registered

Miscellaneous reasons

No reason given

\section{Nonregistrants}

(percent)

21

12

8

6

5

4

4

2

2

28

8

${ }^{8}$ Proportion of interviewed people who registered (68 percent) is higher than the estimated overall registration rate (65 percent) because the latter is a weighted average. Rates were below average in some lightly sampled strata and high in some heavily sampled strata, accounting for the discrepancy between raw and weighted figures. 


पी और

-

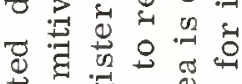

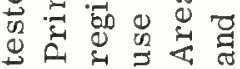

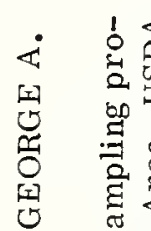

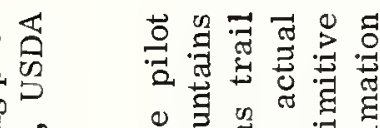

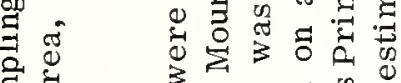

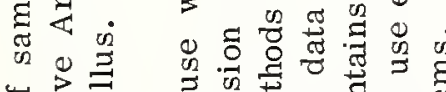
की

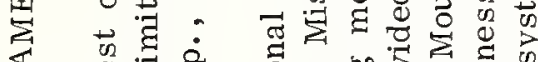

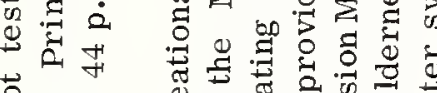

चี

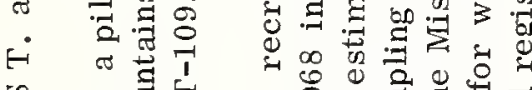

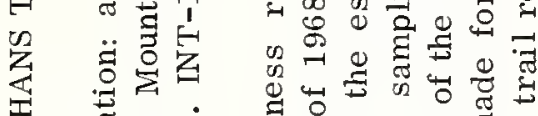

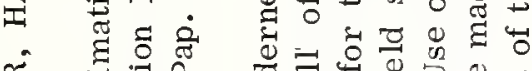

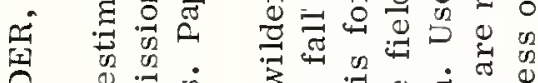

诰

舅

क

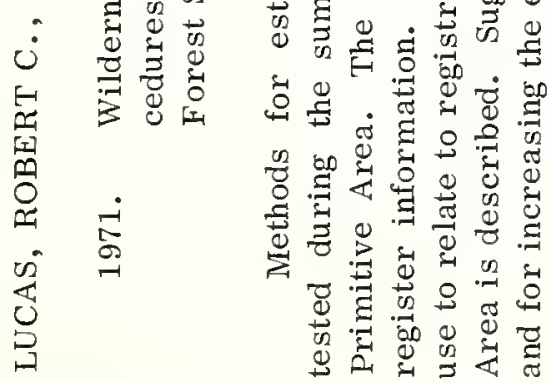

\&

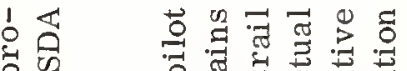

员

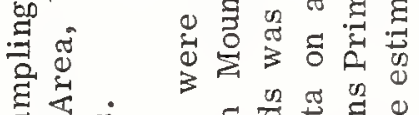

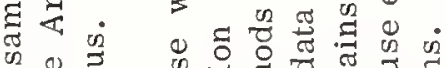

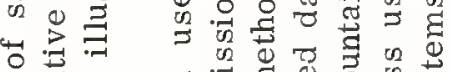

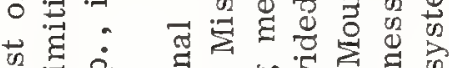

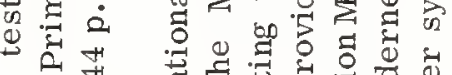

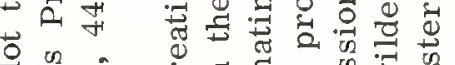

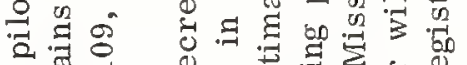

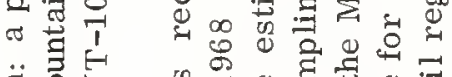

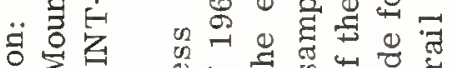

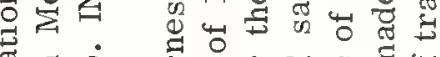

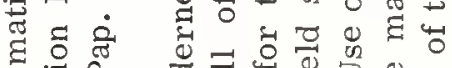

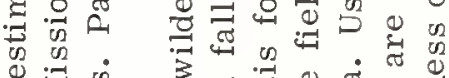

\&

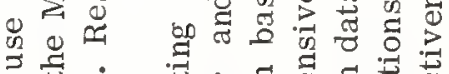

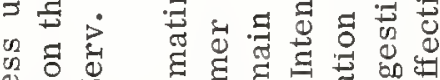

2.

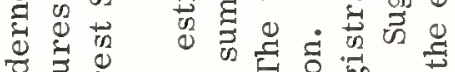

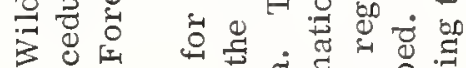

का है 0 음

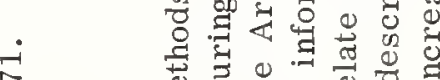

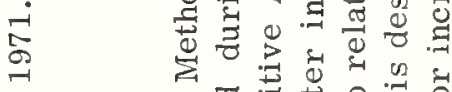
马्य 
So little time was required to register that perhaps those unwilling to take the time didn't understand the importance of registering.

The field interviewers were convinced that at least a majority of the people who said they planned to register when leaving were telling the truth, rather than rationalizing. If this is the case, then the registration rates reported above might be consistently 2 or 3 percentage points low.

The people who said they did not see the box (5 percent) were also probably truthful. We watched several people, usually bent over with heavy packs, walk within 5 feet of the registration station without seeming to notice it at all.

\section{Comparison With Results of Previous Studies}

Many of our findings can be compared quite directly to those reported by Wenger and Gregersen (1964) (see table 2). Very few comparisons with the results reported by Thorsell (1968) are possible, except for those dealing with registration rates (about twice as high in the Mission Mountains Primitive Area) and variations in such rates from trail to trail, (which were less in our study). Comparisons are important because they suggest the degree to which the same factors influence registrations in the same ways in different wildernesses, and they indicate whether the same use-estimating formula could be applied in different areas.

Although Wenger and Gregersen did not publish confidence intervals for their estimates, it seems unlikely that the difference between the overall registration rates in the Oregon study and in our study is statistically significant. The information visitors provided as to their addresses, times of entry and departure, and group sizes was slightly more complete in our study than that reported in the Oregon study.

Most factors seem to influence registration rates in the Oregon study as well as in our study, although usually to a lesser degree in our study. However, the effects of two factors--fishing and the time of the week--were reversed. Unfortunately, the fishing classification was the one factor that was not defined in the same way in the two studies. In Oregon, visitor groups were classified as "fishermen" only if the specific purpose of the trip was fishing for the entire group. In Montana, they were classified as "fishermen" if some of the group fished or planned to fish. 


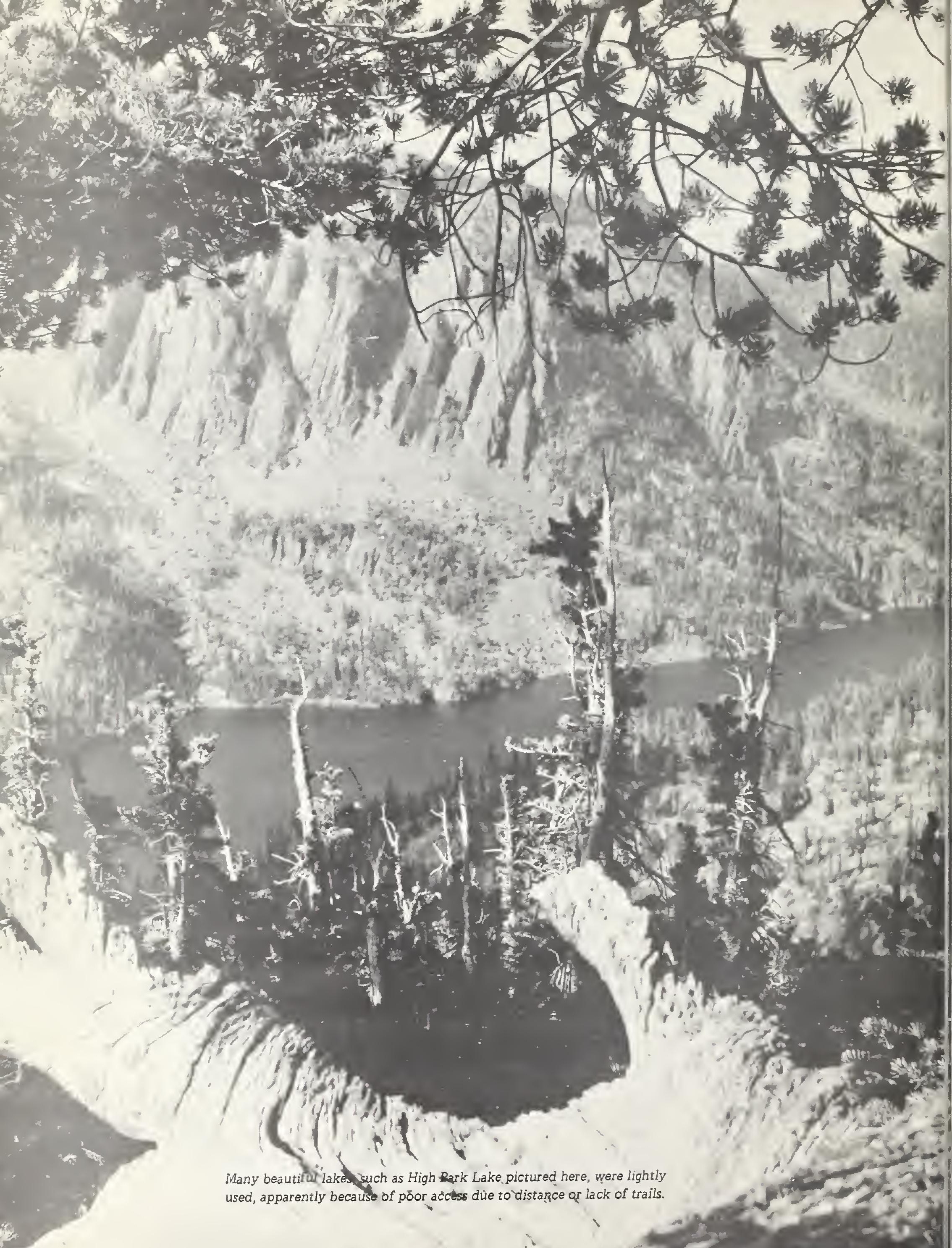




\section{GENERATION OF USE ESTIMATES}

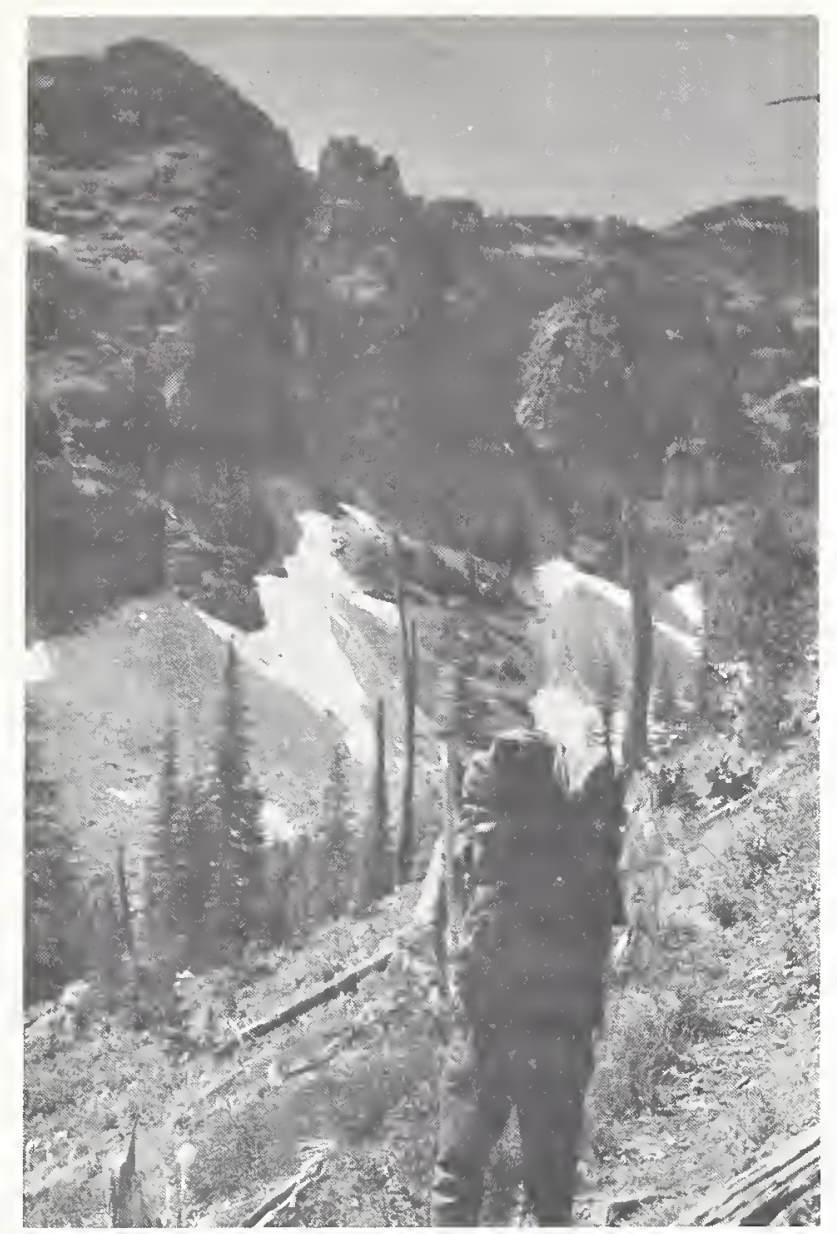

Regression estimates of use were not improved by adding information on one-and two-person and local groups.

The basic data from which use estimates were generated included information obtained from registration cards completed by visitors entering the area (called $\mathrm{X}$ variables); personal interviews on sampling days with entering visitors after they had had an opportunity to register at the registration stations ( $Y$ variables); and personal interviews on sampling days with visitors leaving the area ( $Z$ variables). Several analytical estimation procedures were tested to determine which one produced the most precise estimates of use including: (a) registration information, (b) ratio estimation, (c) stratified random-sampling estimation, and (d) simple and multiple regression estimation.

The target (or planned) population we wanted to estimate included all visitors to the Mission Mountains Primitive Area. However, the sampled populations on which analyses were based were slightly different from this target population because we didn't sample: (a) The few winter and spring visitors, (b) users of lightly used and unknown trails, (c) visitors who did not enter on established trails, and (d) users who entered and left on sampling days during late evening and early morning hours (from $8 \mathrm{p.m}$. to $8 \mathrm{a} . \mathrm{m}$.$) . Some indication of the effect of our decision not to sample during$ these hours is the fact that about 11 percent more groups were interviewed upon leaving than upon entering (412 vs. 370 groups). This was due primarily to early entry of visitors into the area. A few groups probably entered so early and left so late that they were missed entirely. In addition, some uses such as total use in visitor-days were estimated in terms of how long people expected to stay rather than how much time they actually spent in the area.

Estimates of use were generated for 13 variables (see Appendix III), only three of which developed as variables of primary importance. These three (called key variables throughout this report) are (a) number of visitors ( $i=1$ ), (b) use in 
visitor-days $(i=6)$, and $(c)$ number of groups $(i=7)$. Other variables--number of riding groups, saddle animals, pack animals, and so forth--were of secondary importance because almost all use of the wilderness was by hikers.

The relative strengths and weaknesses of each of the use-estimation procedures are discussed along with the estimates derived on pages 24-34.

\section{Registration Station Data}

A box containing registration forms together with a sign requesting each entering group to register is the least expensive technique for obtaining information about wilderness users. Used alone, however, it cannot provide reliable estimates of recreation use, mainly because of nonregistration by some groups. At best, the method can provide lower bounds to estimated use. It can tell an administrator that an area is receiving at least as much use as the registration totals indicate, assuming (a) that entering groups furnish reasonably accurate answers to all questions, and (b) that there is little stuffing of registration boxes with falsely completed forms. These seemed to be safe assumptions. Information derived from uptrail interviews confirmed the accuracy of answers on registration cards and also revealed that only two groups out of 470 "stuffed" the boxes.

Information from all completed registration forms was added to produce raw, uncorrected estimates of use by strata, for all variables (see table 4 for key variables and table 10, Appendix III, for all variables).

Table 4.--Estimates of recreation use on the Mission Mountains Primitive Area, Montana, based on row (uncorrected) registration information, summer-fall, 1968

\begin{tabular}{|c|c|c|c|c|c|c|}
\hline Strata & $:$ & Visits & $\begin{array}{l}: \\
:\end{array}$ & Total use & : & Groups \\
\hline & & Number & & Visitor-hours 1 & & Number \\
\hline Summer & & 2,705 & & 30,265 & & 680 \\
\hline Fall & & 354 & & 4,527 & & 130 \\
\hline Summer + Fall & & 3,059 & & 34,792 & & 810 \\
\hline 1 & & 658 & & 6,511 & & 166 \\
\hline 2 & & 597 & & 4,533 & & 169 \\
\hline 3 & & 570 & & 3,932 & & 137 \\
\hline 4 & & 880 & & 15,289 & & 208 \\
\hline 5 & & 140 & & 1,397 & & 51 \\
\hline 6 & & 1.36 & & 2,375 & & 53 \\
\hline 7 & & 78 & & 755 & & 26 \\
\hline
\end{tabular}

${ }^{1}$ Divide visitor-hours by 12 to get visitor-days, as defined for federal recreation area statistics. 


\section{Ratio Estimation [Corrected Registration Information]}

To generate more accurate estimates of use from raw data computed from completed registration forms, the data can be corrected using an expansion factor based on the estimated registration rate; in statistical terms, this provides a ratio estimate. ${ }^{9}$ Estimates of use with their associated standard errors, corrected for registration rate, are shown in table 5 for the key variables.

Raw registration totals were multiplied by the following correction factors: number of visits, 1.35 ; number of visitor-hours, 1.63 ; and number of groups, 1.24 .

Table 5.--Estimates of recreation use on the Mission Mountains Primitive Area, Montana, based on ratio estimation (corrected registration information), summer-fall, 1968

\begin{tabular}{|c|c|c|c|c|c|c|c|c|}
\hline \multirow{2}{*}{ Strata } & : & \multicolumn{2}{|c|}{ Visits } & \multicolumn{2}{|c|}{ : $\quad$ Total use } & \multirow[b]{2}{*}{ : } & \multicolumn{2}{|c|}{ Group } \\
\hline & ${ }^{\circ}$ & Estimate & $\begin{array}{l}: \text { Standard } \\
: \text { error }\end{array}$ & : Estimate & $\begin{array}{l}: \text { Standard } \\
: \text { error }\end{array}$ & & Estimate & $\begin{array}{l}: \text { Standard } \\
: \text { error }^{1}\end{array}$ \\
\hline & & Number & Percent & Hours & Percent & & Number & Percent \\
\hline Summer & & 3,652 & 6.3 & 49,332 & 14.7 & & 843 & 8.1 \\
\hline Fal1 & & 478 & do. & 7,379 & do. & & 161 & do. \\
\hline Summer + Fal1 & & 4,130 & do. & 56,711 & do. & & 1,004 & do. \\
\hline 1 & & 888 & do. & 10,613 & do. & & 206 & do. \\
\hline 2 & & 806 & do. & 7,389 & do. & & 210 & do. \\
\hline 3 & & 770 & do. & 6,409 & do. & & 170 & do. \\
\hline 4 & & 1,188 & do. & 24,921 & do. & & 258 & do. \\
\hline 5 & & 189 & do. & 2,277 & do. & & 63 & do. \\
\hline 6 & & 184 & do. & 3,871 & do. & & 66 & do. \\
\hline 7 & & 105 & do. & 1,231 & do. & & 32 & do. \\
\hline
\end{tabular}

${ }^{1}$ Standard error, expressed as percentage of the estimate. Standard errors do not vary from stratum to stratum because in ratio estimation variance is unconditional.

${ }^{9}$ We used the "combined ratio estimate" from Cochran (1963, section 6.11). 
Ratio estimates also have advantages from a statistical standpoint. The ratio estimates had the smallest error terms of any of the estimation methods tested. A ratio estimate is based on an as sumption of linearity through the origin--in other words, when there are no registrations, there would be no estimated use (Cochran 1963, section 6.9). This assumption fits well. We observed use only for 12 hours on each of the 2 days in a sample unit, while registered use was measured over the whole 48-hour period. This means registered use could be either larger or smaller than observed use. If use and registration were measured over identical time periods, actual use could not be less than registered use. This difference could introduce a bias, probably small.

Ratio estimation is also best if the variance of $Y$ at a given value of $X$ is proportional to the value of $X$--in other words, if the variance of $Y$ increases as $X$ increases. This also seems reasonable for trail registration data.

In practical terms, ratio estimation is easy for land managers to understand and apply. The procedure is intuitively obvious and the calculations are very simple.

\section{Stratified Random-Sampling Estimation}

Unbiased estimates of use with their associated standard errors were generated using stratified random-sampling estimation procedures (table 6). Two sets of estimates were produced: one based on data from persons interviewed when entering, the other based on persons interviewed when leaving. These data were multiplied by the inverse of the sampling fraction. For example, 12 of 14 possible 2-day units were sampled in stratum 1 , so the interview totals were multiplied by $14 / 12$ or 1.1667 .

The estimates of the number of visits and number of groups entering and leaving were about the same in the summer, but were markedly different in the fall. This was attributed to the fact that visitors who entered the area prior to the arrival of the interviewer at $8 \mathrm{a} . \mathrm{m}$. (or at 12 noon the last few weeks) were more common in the fall than in the summer.

Expected and actual hours of use were quite similar in the summer, but expected use $(12,996$ hours) far exceeded actual use $(3,681$ hours) in the fall. This was partly attributed to the fact that unplanned factors reduced actual length of stay in the fall about 10 percent below visitors' expectations. However, most of the difference between expected and actual hours of use in the fall was attributed to sampling variability in stratum 7 (one group that planned to stay 8 days--an unusually long time--was interviewed only upon entering). The precision of total estimated summer visitor-hours was high for both entering and exiting visitors, but was unacceptably low for total estimated fall visitor-hours. More precise estimates for the three key variables were obtained by combining summer and fall strata.

Sampling to obtain estimates of use was far less efficient in the fall than in the summer. This probably reflected the light and variable use in the fall. It is also more difficult to select hours in the fall for interviewing that would assure contacting nearly all visitors at least once. Some hunters enter early in the day, a few leave late.

This type of estimation did not give adequate precision at reasonable cost. Sampling intensities during summer, fal1, and summer+fall were high (13, 15, and 14 percent, respectively) as were costs; yet precision levels for several variables were considerably less than desired. One advantage of this method is that unbiased estimates and precision levels are obtained for all variables of interest.

Where highly reliable estimates are obtained for several variables, as was the case for some strata in this study, they can be used as a standard for comparison with estimates from other estimation methods. These other methods might be more efficient, 
but they depend on certain assumptions that cannot be adequately tested. Such a comparison would only have validity if "loopholes" were closed, such as the visitors who entered or left on sample days when the interviewers were not on the trail. Otherwise the sampling fraction, based on the proportion of days sampled, exaggerates the proportion of use sampled. One way of dealing with this problem would be to define the sampling frame in terms of hours, and choose blocks of hours randomly. The extremely late and early hours, say from 1l:00 p.m. to 5:00 a.m., could probably be safely ignored.

Table 6.--Stratified random-sampling estimates of recreation use on the Mission Mountains Primitive Area, Montana, based on interview of entering and leaving visitors, summer-fall 1968

\begin{tabular}{|c|c|c|c|c|c|c|c|c|}
\hline \multirow{2}{*}{ Stratum } & \multirow{2}{*}{$\begin{array}{l}: \\
\vdots \\
: \\
:\end{array}$} & \multicolumn{3}{|c|}{ Visits } & \multicolumn{2}{|c|}{ Total use } & \multicolumn{2}{|c|}{$: \quad$ Groups } \\
\hline & & \multirow{2}{*}{$\frac{\text { Estimate }}{\text { Number }}$} & \multicolumn{2}{|c|}{$\begin{array}{l}\text { :Standard } \\
: \text { error }^{1}\end{array}$} & Estimate & $\begin{array}{cc}: & \text { Standard } \\
: & \text { error }^{1}\end{array}$ & \multirow{2}{*}{$\frac{: \text { Estimate }}{\text { Number }}$} & \multirow{2}{*}{$\begin{array}{cc}: & \begin{array}{c}\text { Standard } \\
\text { error }\end{array} \\
& \text { Percent }\end{array}$} \\
\hline & & & & Percent $V$ & Visitor-hou & Percent & & \\
\hline & & BASED & $\mathrm{ON} 1$ & INTERVIEW & OF VISITOR & S UPON ENTRAI & NCE & \\
\hline Summer & & 2,878 & & 11.3 & 20,918 & 12.9 & 748 & 10.3 \\
\hline Fall & & 506 & & 22.9 & 12,996 & 59.8 & 209 & 18.9 \\
\hline Summer + Fall & & 3,384 & & 10.6 & 33,914 & 24.3 & 957 & 9.0 \\
\hline 1 & & 763 & & 6.8 & 6,795 & 13.2 & 190 & 5.8 \\
\hline 2 & & 692 & & 11.3 & 5,619 & 17.6 & 209 & 8.6 \\
\hline 3 & & 686 & & 16.9 & 3,774 & 23.7 & 160 & 17.5 \\
\hline 4 & & 737 & & 41.5 & 4,730 & 46.0 & 189 & 36.0 \\
\hline 5 & & 228 & & 37.7 & 958 & 26.3 & 97 & 33.0 \\
\hline 6 & & 200 & & 31.0 & 4,039 & 44.6 & 83 & 25.3 \\
\hline \multirow[t]{2}{*}{7} & & 78 & & 60.3 & 8,154 & 92.7 & 29 & 41.4 \\
\hline & & BASED & $\mathrm{ON}$ & INTERVIEW & W OF VISITO & RS UPON DEPAI & RTURE & \\
\hline Summer & & 2,898 & & 12.1 & 22,275 & 8.0 & 776 & 9.6 \\
\hline Fall & & 668 & & 20.4 & 3,681 & 25.0 & 273 & 19.3 \\
\hline Summer + Fall & & 3,566 & & 10.6 & 25,956 & 7.8 & 1,049 & 8.7 \\
\hline 1 & & 838 & & 6.8 & 8,038 & 9.9 & 209 & 5.6 \\
\hline 2 & & 600 & & 13.8 & 4,125 & 11.5 & 191 & 10.5 \\
\hline 3 & & 837 & & 16.7 & 7,281 & 14.8 & 206 & 16.9 \\
\hline 4 & & 623 & & 49.1 & 2,832 & 38.4 & 170 & 36.0 \\
\hline 5 & & 215 & & 40.0 & 652 & 37.3 & 93 & 38.2 \\
\hline 6 & & 297 & & 22.2 & 2,279 & 35.4 & 131 & 22.1 \\
\hline 7 & & 156 & & 52.6 & 750 & 49.9 & 49 & 52.5 \\
\hline
\end{tabular}

${ }^{I}$ Standard error, expressed as percentage of the estimate. 


\section{Regression Estimation}

Estimates of use and associated standard errors were generated for the three key variables using simple linear regression estimation procedures. The three basic regression equations used in this analysis expressed linear functions of the corresponding covariate.

The precision of total estimated summer use was high for all three key variables, but unacceptably low for all fall-use estimates (table 7). The reasons for the large standard error terms for all variables in the fall cannot be determined precisely. Sampling intensity should have been adequate with a 29-, 14-, and 9-percent sampling rate, respectively, in strata 5, 6, and 7 . A zero registration rate likely accounted for the low precision level in stratum 7, yet registration rates of 58 and 57 percent were obtained for strata 5 and 6 , respectively. The high standard errors may be a function of the greater inherent variability of fall use and registration rates. The average length of stay was a little longer during the fall than it was during summer, but it was much more variable. Such use included (a) a mixture of deer and elk hunters walking the lower elevation trails for a few hours, (b) fishermen and hikers out for the day, and (c) mountain goat hunters camping for days in the high country.

Summer and fall strata were pooled to generate season-long estimates of use for the key variables using simple linear regression estimation methods (table 8). The advantage of pooling all strata, of course, is the large sample size obtained ( $n=110$ 2-day sample units). Fairly precise season-long estimates of use were obtained for all variables. Pooling all strata can, of course, yield misleading results if some of the strata are substantially different populations.

Table 7.--Regression estimates of recreation use on the Mission Mountains Primitive Area, Montona, based on self-registration--interview relationships, sumer-falI, 1968

\begin{tabular}{|c|c|c|c|c|c|c|}
\hline \multirow{2}{*}{ Stratum } & \multicolumn{2}{|c|}{ Visits } & \multicolumn{2}{|c|}{ Total use } & \multicolumn{2}{|c|}{ Groups } \\
\hline & Estimate & $\begin{array}{l}\text { Standard } \\
: \text { error }^{1} \\
\end{array}$ & $:$ Estimate & $\begin{array}{lc}: & \text { Standard } \\
: & \text { error } \\
\end{array}$ & $\begin{array}{l}: \text { Estimate } \\
\vdots\end{array}$ & $\begin{array}{l}\text { : Standard } \\
: \text { error } \\
\end{array}$ \\
\hline & Number & Percent & Visitor-hours & Percent & Number & Percent \\
\hline Summer & 3,622 & 8.9 & 39,544 & 13.9 & 910 & 10.0 \\
\hline Fal1 & 634 & 49.0 & 8,046 & 59.8 & 220 & 36.7 \\
\hline Summer + Fal1 & 4,256 & 10.4 & 47,590 & 17.7 & 1,130 & 13.7 \\
\hline 1 & 836 & 4.2 & 7,976 & 7.1 & 208 & 5.3 \\
\hline 2 & 765 & 5.5 & 5,666 & 12.0 & 214 & 6.1 \\
\hline 3 & 739 & 7.2 & 5,036 & 17.0 & 176 & 8.5 \\
\hline 4 & 1,282 & 22.3 & 20,866 & 21.3 & 312 & 23.7 \\
\hline 5 & 199 & 28.6 & 2,004 & 48.0 & 70 & 22.8 \\
\hline 6 & 277 & 68.2 & 4,314 & 102.5 & 99 & 49.5 \\
\hline 7 & 158 & 72.8 & 1,728 & 112.4 & 51 & 59.0 \\
\hline
\end{tabular}

${ }^{1}$ Standard error, expressed as percentage of the estimate. 
Tab1e 8.--Regression equations, coefficients of determination ( $\left.R^{2}\right)$, and standard errors for the key variables

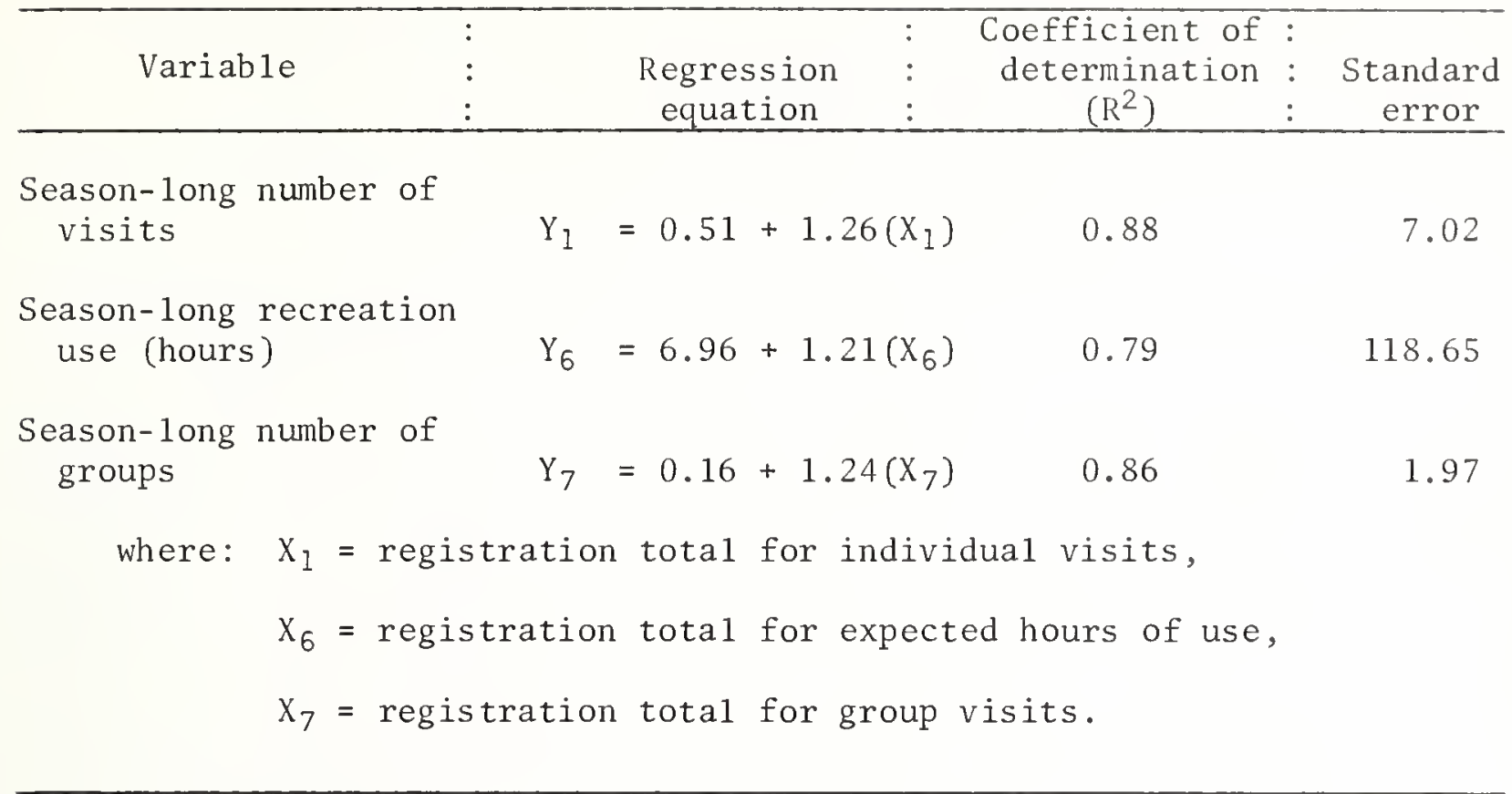

Multiple regression analyses were next run to determine whether additional covariate information might result in more precise estimates of use, especially for the fall strata. Variables representing one- and two-person groups and local groups were added as covariates. These additional covariates were included because earlier work by Wenger and Gregersen (1964) indicated that small groups and local groups tended to register at lower rates than other groups. Results indicated that although the data for these types of use based on interviews generally improved precision as measured by the standard error of estimate, substitution of registration data for interview data essentially wiped out this improvement. Estimating procedures using interview data don't seem practical because an administrator would ordinarily have to work with registration data.

Regressions were also run to determine whether numbers of registered users might also give an adequate estimate of hours of use and number of groups. It was found that registered visits could be used as well as registered groups in the prediction of total number of groups, but the registered visits variable was considerably poorer than registered visitor-hours in the prediction of total visitor-hours.

\section{Mechanical Counters}

Mechanical counters were used in conjunction with interview information to evaluate devices that might be used for a several-year period following calibration to obtain inexpensive and acceptably accurate estimates of use. The study was concerned mainly with evaluating counting devices, rather than attempting to establish prediction equations based on their results. 
Table 9.--Regression equations, coefficients of determination ( $R^{2}$ ), and standard errors for the key variables, based on mechanical counter relationships

\begin{tabular}{|c|c|c|c|}
\hline Variable & $\begin{array}{l}\text { Regression } \\
\text { equation }\end{array}$ & $\begin{array}{l}\text { Coefficient of } \\
\text { determination } \\
\left(R^{2}\right)\end{array}$ & $\begin{array}{l}\text { Standard } \\
\text { error }\end{array}$ \\
\hline \multicolumn{4}{|c|}{ VEHICLE TRAFFIC-COUNTER REGRESSION } \\
\hline $\begin{array}{l}\text { Season-long number of } \\
\text { visits }\end{array}$ & $Y 1=-3.63+1.29(\mathrm{TC})$ & 0.67 & 17.68 \\
\hline $\begin{array}{l}\text { Season-long recreation } \\
\text { use (hours) }\end{array}$ & $Y 6=-1,596.40+148.79$ & $(\mathrm{TC})$ & $3,855.29$ \\
\hline $\begin{array}{l}\text { Season-long number of } \\
\text { groups }\end{array}$ & $Y 7=0.47+0.29(\mathrm{TC})$ & .66 & 4.16 \\
\hline & TRAIL PRESSURE-PAD COUNTEF & R REGRESSION & \\
\hline $\begin{array}{l}\text { Season-long number of } \\
\quad \text { visits }\end{array}$ & $Y 1=9.33+0.84(\mathrm{PC})$ & .83 & 14.85 \\
\hline $\begin{array}{l}\text { Season-long recreation } \\
\text { use (hours) }\end{array}$ & $Y 6=2.12+104.21(P C)$ & .48 & $4,145.85$ \\
\hline $\begin{array}{l}\text { Season-long number of } \\
\text { groups }\end{array}$ & $Y 7=5.20+0.16(P C)$ & .69 & 4.00 \\
\hline
\end{tabular}

The prototype ultrasonic beam counters could not be kept in adjustment and operating long enough to yield any usable counts. The counters operated well when tested indoors, but failed in the field, probably due to temperature fluctuations and moisture. The trail pressure-pad counter and traffic counters worked quite satisfactorily, however, and yielded useful information.

The regression equations expressed interview information obtained from entering visitors for the three key variables as linear functions of the corresponding pressurepad counter (P.C.) and traffic counter (T.C.) covariate values (table 9). The analysis revealed that trail pressure-pad counters and vehicular traffic counters hold promise in estimating number of persons and number of groups, but that mechanical counters were essentially useless in estimating total visitor-hours. The single trail pressurepad counter provided more precise estimates of number of persons and groups than did vehicular traffic counters on two access roads. However, both types of counters were inferior to registration boxes in estimating use. 


\section{Summary of Use Estimates}

A summary of use estimates for key variables, based on results of ratio estimation is shown below for the Mission Mountains Primitive Area for the period June 15 to November 11, 1968, inclusive:

variable

Total season-long visits

Number of summer visits

Number of fall visits

Total season-long group visits

Number of summer group visits

Number of fall group visits

Total season-long visitor-hours

Total season-long visitor-days 11

Summer visitor-hours

Summer visitor-days 11

Fall visitor-hours

Fall visitor-days 11

Estimate
4,130
3,652
478
1,004
843
161
56,711
4,726
49,332
4,111
7,379
615

$\frac{\text { Standard errorio }}{\text { (Percent) }}$

6.3

6.3

6.3

8.1

8.1

8.1

14.7

14.7

14.7

14.7

14.7

14.7

The average length of stay was about 14 hours, and the average group size was 4.1 persons. Over 80 percent of all groups left the wilderness the same day they entered. Such a short average stay is much less than generally expected by recreation planners and managers for wilderness users, but it agrees with the results of studies in Oregon by Wenger (1964) and in the Boundary Waters Canoe Area by Lucas (1964). Another study in three Oregon and Washington wildernesses (Hendee et al. 1968) shows stays averaging on 1 y 2.2 to 3.0 days.

More than 94 percent of the visitors were estimated to be hikers. Most of the very limited horse use occurred in the fall. Hikers accounted for 97 percent of the summer use and 85 percent of the fall use. Only one user in a hundred was estimated to have employed guides or outfitters.

Use was distributed very unevenly throughout the Mission Mountains Primitive Area. This agrees with all previous studies of wilderness use. The estimated season-long distribution of use is shown in figure 8. Forty-four percent of all groups entered the area along the Glacier Lake Trail. Another 18 percent entered along the Cold Lakes Trail. Lakes appeared to be the major attraction, but use was appreciable only where there were trails leading to them. Some large lakes were lightly used, apparently because of long distances involved or because of lack of trails to them. Use dropped rather quickly as distance from access points increased.

This use distribution was rather different than the prestudy trail use estimates made by the administrators of the area for planning stratification. The summer pattern was more extreme; a few places were more heavily used than estimated, and a great many were more lightly used. In the fall, Glacier Lake was used more heavily than had been estimated on weekends, but almost all other locations were used less than expected.

${ }^{10}$ Standard error, expressed as percentage of the estimate.

${ }^{11} \mathrm{~A}$ visitor-day is defined as a recreation use that aggregates 12 person-hours. It might entail one person for 12 hours, 12 persons for 1 hour, or any equivalent combination of individuals or group use, either continuous or intermittent. 


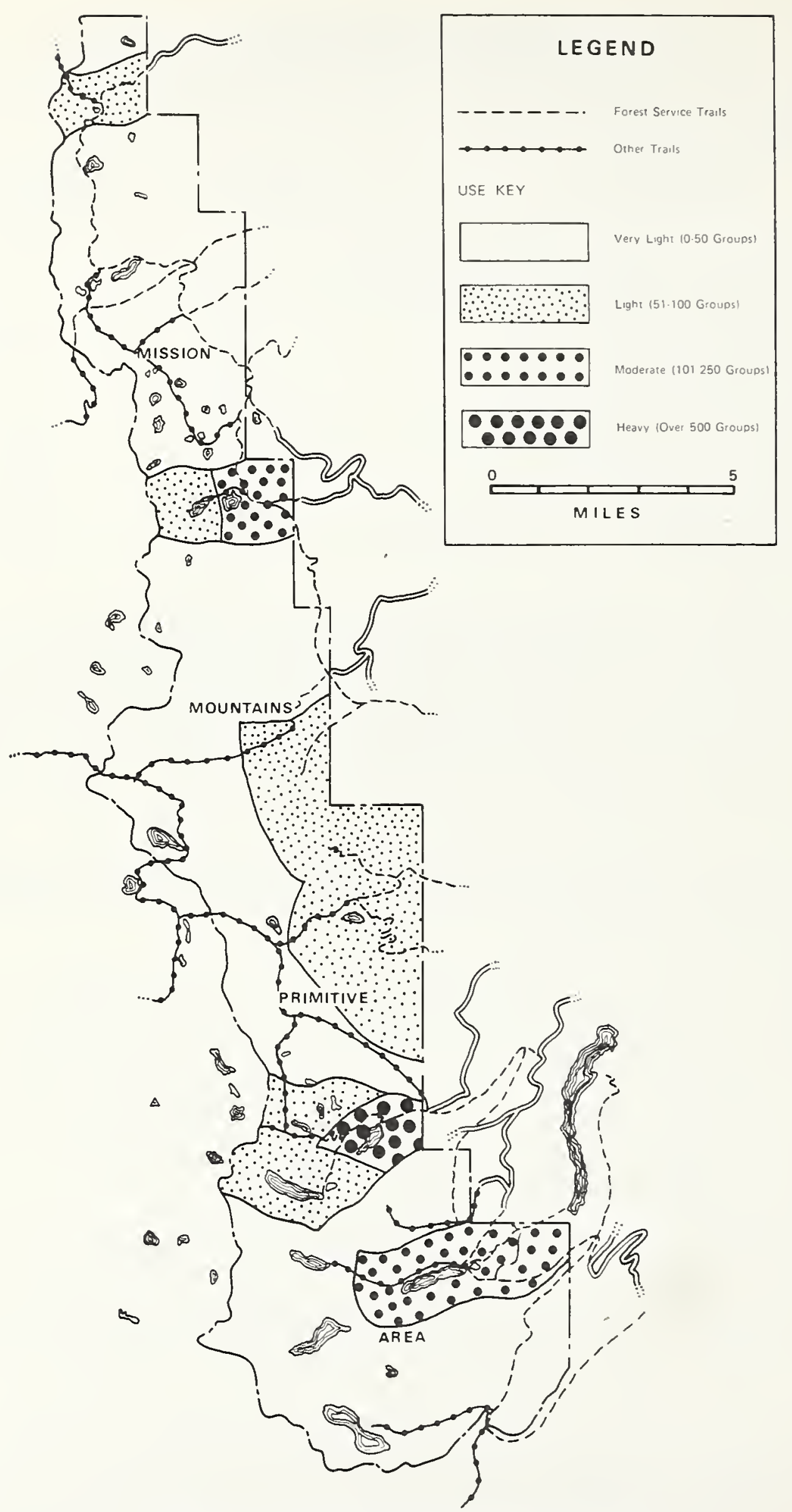

Figure 8. - Map showing season-long distribution of estimated use on the Mission Mountains Primitive Area. 
Seventy-eight percent of all interviewed groups were residents of Montana, 92 percent of whom lived more than $60 \mathrm{miles}$ from the Missions. Origin for out-of-State visitors follows:

\section{Percent of total use}

$\begin{array}{ll}\text { California } & 3 \\ \text { Minnesota } & 2 \\ \text { Washington } & 2 \\ \text { Illinois } & 1 \\ \text { Oowa } & 1 \\ \text { Oregon } & 1 \\ \text { Wisconsin } & 1 \\ & 3 \\ \text { Michigan, Indiana, } & 3 \\ \quad \text { Ohio, Kentucky } & 1 \\ \text { Northeastern States } & 1 \\ \text { Rocky Mountain \& Intermountain States } & 1 \\ \text { Southeastern States } & \\ \text { Nebraska, Kansas, Missouri } & \end{array}$

Four parties from Europe visited the area during 1968.

Fishing was the major activity in terms of time spent, accounting for an estimated 38 percent of all visitor-hours reported by parties leaving the area. Hiking was second, accounting for 26 percent; followed by camping, 16 percent; hunting, 10 percent; nature study, 3 percent; and miscellaneous activities, approximately 7 percent. Mountain climbing accounted for only $1 / 2$ of 1 percent of total visitor-hours.

In summary, the Mission Mountains Primitive Area appears to be predominantly a summer, hiking, day-use wilderness. Use is distributed very unevenly within the area. It enjoys a largely regional clientele, but vacationists from all over the United States visit the area.

\section{Cost of Using Same Estimation Technique}

What might it cost to use the same estimation technique in the future? Cost of the pilot study was $\$ 11,500$ for all field-sampling phases; approximately $\$ 1,500$ for analyses and report preparation. A breakdown follows:

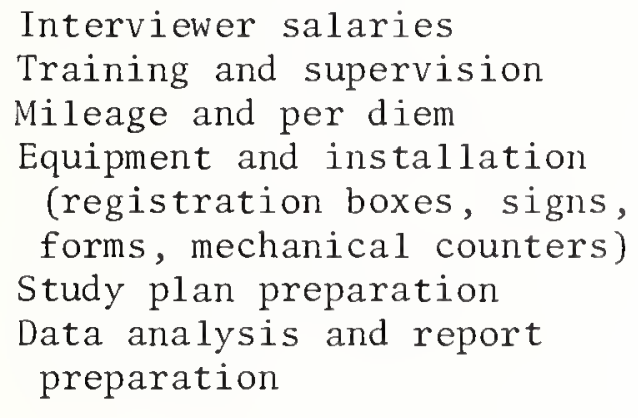

Total

$$
\begin{array}{r}
\$ 7,700 \\
600 \\
1,500 \\
\\
700 \\
1,000 \\
1,500 \\
\hline
\end{array}
$$

$\$ 13,000$

The field-sampling cost $(\$ 11,500)$ can be prorated over several years because of relatively strong relationships between registration and interview information. Assuming that relationships between use and registration information remain constant, 
estimates of use can be updated annually for perhaps 3 to 4 years based on selfregistration information only, without interviewing entering visitors. It would be necessary only to solve the regression equation or apply the ratio estimate "correction factors" using registration information obtained each year. Thus, the average annual cost would amount to approximately $\$ 3,000$, including about $\$ 300$ annually for servicing registration stations, travel, and administration, which managers would probably have to spend anyway. The same registration system should continue in use for the period of time that use estimates are updated in this manner. 


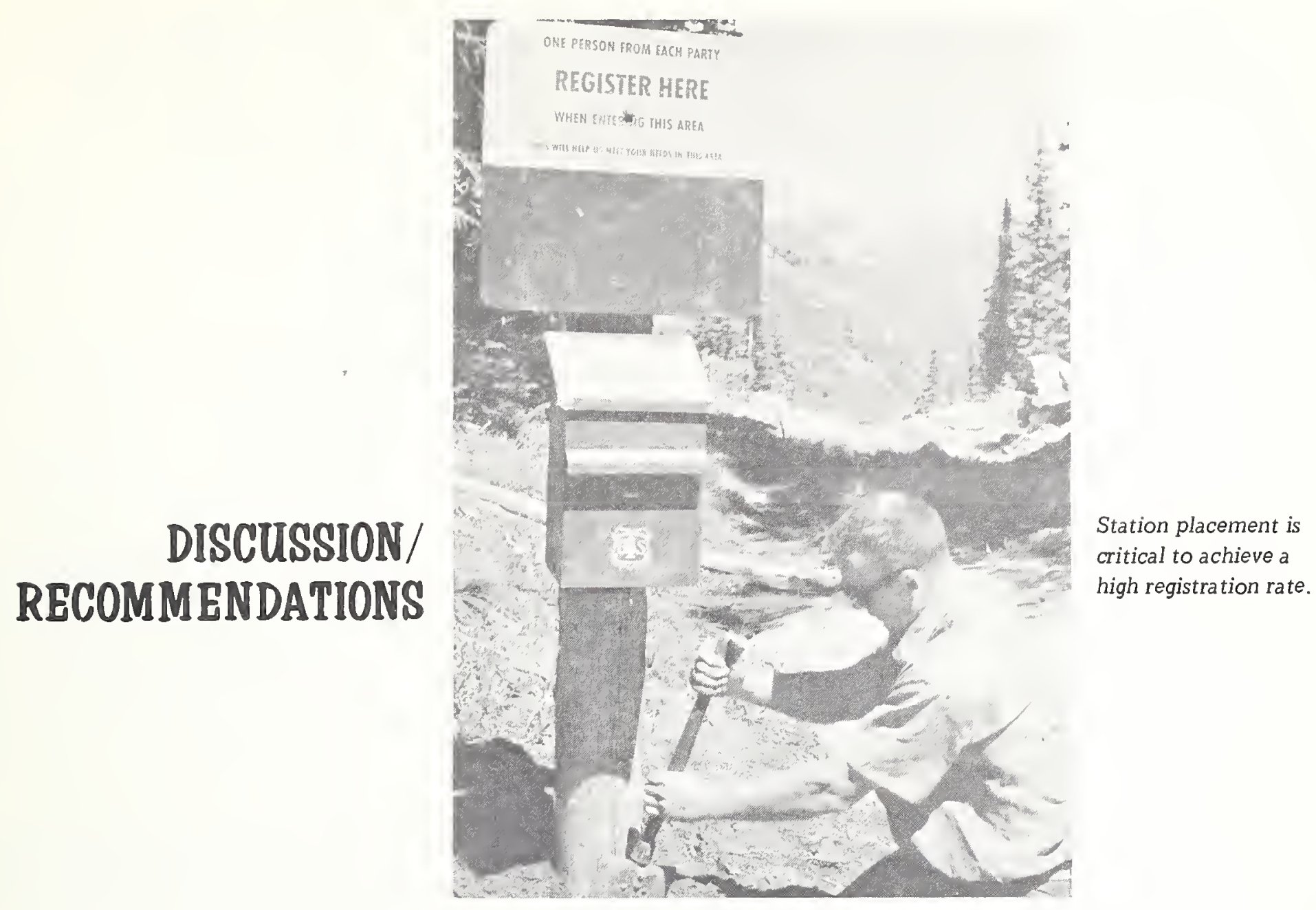

The study resulted in the development of a useful sampling tool for estimating current recreation use. This "tool" provides a means of updating use estimates for several years following field sampling. However, the sampling model is not recommended for general use because of high costs and certain weaknesses that must yet be corrected. The study yielded information that should make it possible to substantially reduce costs and improve sampling efficiency in future studies.

\section{Sample Design}

Sampling costs can be minimized by sound selection of sample size and allocation of sampling effort. Depending upon the characteristics of the population to be sampled, stratification may result in a gain in precision of estimates. However, stratification is unnecessary and complicates calculations where the population is homogeneous.

The most serious weakness in our sampling model was in the allocation of sampling effort. The sampling effort was subjectively allocated disproportionately between strata, but the analysis revealed that sample allocation was not optimum. In terms of maximum sampling efficiency and precision of estimates, some strata were sampled too heavily while other strata were not sampled heavily enough. Sampling effort should have been concentrated in the moderate-to-heavy-use strata, and little or no sampling should have been done in the light-use strata. 
Each group interview on lightly used trails cost approximately $\$ 100$, compared to a cost of only $\$ 5$ per interview on heavily used trails, because few interviews were obtained per sampling unit. For example, stratum 4 (containing many lightly used trails) yielded only 12 interviews during 36 days of interviewing; stratum 7 yielded only 7 interviews during 22 days of interviewing. The least-used third of all trails yielded less than 5 percent of all registrations. Deletion of these trails from the sampling frame would have resulted in only a slight loss in the precision of estimates and would have cut sampling costs almost in half. Use of lightly used trails could have been adequately estimated by means of registration stations only, without enploying uptrail interviewing.

Elimination of all lightly used trails from the sampling frame would have meant that the large, highly stratified sample would not have been necessary. Relationships between registration data and actual use were fairly similar from trail to trail; variation was small enough that strata representing different trails, seasons, and time of week could be pooled without significant loss of precision of the estimates. Considerably less than the 110 sampling units used in the study would have been expected to yield use estimates of acceptable precision.

A presampling method is needed to determine optimum stratification and to identify lightly used trails. Installing registration stations on all trails 1 year prior to field sampling would likely provide a satisfactory screening method. Although all entering groups do not register, the completed registration forms would indicate the relative magnitude of use on each trail.

\section{Best Estimation Procedures}

Both linear regression and ratio estimate (correction factor) procedures yielded acceptably accurate estimates. However, the ratio estimates were somewhat more precise (had smaller error terms), and would generally be easier for administrators to apply.

\section{Extension of Results to Other Wildernesses}

The prediction equations or correction factors generated in this study are not applicable to other unsampled wildernesses. Yet, our results were quite similar to those obtained by Wenger and Gregersen on the Three Sisters and Mountain Lakes Wildernesses in Oregon. The similarity in registration-use relationships for these three areas suggests that more economical ways of estimating use and establishing relationships between use and registration might be found. It is possible that particular types of wildernesses might have similar registration rates and use patterns. Other day-use riking areas might also prove to be fairly similar. If further studies confirm this hypothesis, it might be possible to sample registration responses at one wilderness area only enough to confirm an expectation based on the type of wilderness involved.

Furthermore, it might also turn out that relationships change little over time. The general similarity in use patterns on the Mission Mountains Primitive Area, which was sampled during 1968, and the Oregon wildernesses, which were sampled 6 and 7 years earlier, indicates relative stability over time. If so, estimating equations might be used for rather long periods of time, perhaps 5 years or more, and only require limited periodic checking to detect any shifts with time.

\section{Personal Interviews and Automatic Counters}

The largest cost item in this and earlier studies was personal interviewing, which accounted for approximately 75 percent of the total sampling cost. Therefore, we should seek to eliminate or reduce the need for interviewing by devising some substitute methods, such as mechanical or electrical counters or automatic cameras. Although 
trail and vehicular traffic counters yielded use estimates in our study that were not as precise as those obtained using regression and correction-factor estimation techniques, our tests using the counters are encouraging.

Development and testing of improved trail counting devices are needed and recommended. Directional, printout counters, that would record time and direction of movement as well as distinguish between hikers and animals (domestic stock and big game), would be particularly valuable. However, counters must be used in conjunction with other techniques, such as registration stations, for collecting information about the trail user because counters provide only simple counts of passing objects and would be useless for obtaining such information as length of stay. Automatic cameras could provide much more data than counters, but a registration system appears to be essential for obtaining descriptive information, such as length of stay, residence of visitors, etc.

\section{Raising Registration Rates}

More precise use estimates can be expected if the rate of registration can be raised. The main underlying reason for not registering appears to be a misunderstanding by users of the importance of registration information to wilderness management and a failure to see any personal advantage to registration. Registration seems to be perceived by many as a casual, voluntary activity. An educational and informational program to "sell" wilderness users on the need for registering seems needed. The management value and personal safety advantage of registering might be stressed in the message on signs; in map brochures made available for each area; in personal contacts with outdoor clubs and outfitters; in newspapers, and outdoor and conservation magazines; and in conservation education programs.

The optimum solution to increasing registration rates probably would be to make registration mandatory. A registration system would provide the information necessary for improving wilderness planning and management--enough, we feel, to offset any slight inconvenience for visitors it might produce. The Boundary Waters Canoe Area has had mandatory registration for several years, and the Forest Service began requiring registration in National Forest Wildernesses in California during the 1971 season.

Improvements in location and placement of registration stations and signs should be made, particularly if registration remains voluntary. The subdued gray and brown colors of stations and signs used during 1968 did little to attract attention. These standard Forest Service sign colors were intended to harmonize with the forest environment, but they might have harmonized too successfully! Wenger (1964) used more conspicuous yellow and black signs and obtained higher registration rates. In addition, the usual trailhead cluster of three sets of signs on three posts--registration box and sign, trail information sign, and "U-6" regulations prohibiting motor vehicles--probably detracted attention from the registration station. Placing the registration station and sign at some distance from the other signs, and very close to the trail, might be expected to attract more attention.

The effect on registration rates of placing stations at the wilderness boundary rather than at the trailhead cannot be estimated from our data. The registration rate might increase in situations where a fairly long trip is required to reach the boundary and the registration station, especially where the station is at a viewpoint after a steep climb, where many people might welcome an excuse to stop and rest awhile. On the other hand, a station located only a mile or two up a trail might produce a lower rate than a trailhead location, especially for horsemen. Placement of hitch racks at registration stations might encourage higher registration by horsemen. 
Simplicity in the registration form probably is also a key to higher response rates. The registration form should be short and clear. The bare minimum of essential information is all that should be asked for. More detailed information can be obtained when and where needed using inexpensive mail surveys. Much of the information that might be desired, such as route followed, campsites used, activities engaged in, fish caught, or game shot can only be accurately obtained after the trip has been completed. More is lost by driving down registration rates with excessive demands for detail than this detail is worth.

\section{Registration Systems}

Whatever form the ultimate sampling model might take, a registration system seems worthwhile as one of the ingredients for wilderness use estimation. It offers better use figures than crude estimates and intuition might provide. Registration information provides detailed information about the forest user, and provides lower bounds for all variables of interest. Registration information can be expected to provide needed insurance if it develops after field sampling that the prediction equations prove to be no longer valid because of changed visitor-use patterns, registration response, or for other reasons. Self-registration also has a number of other advantages besides providing information to managers. It provides a chance to inform visitors of wilderness rules and regulations. It could be a valuable key to the control or redistribution of use within an area. It could prove useful in emergencies. A good registration system could convey to visitors an impression that the managing agency has a serious, professional concern for the wilderness resource and for the visitors.

Registration data can be of direct value for wilderness research as well as providing a source of sample lists of group leaders for mail questionnaires.

There is universal agreement on the need for improved wilderness management. Good estimates of wilderness use and a better understanding of wilderness users are essential for better management. Therefore, it appears highly desirable that wilderness managers move toward a uniform, comprehensive registration system for wilderness visitors. In addition, continuing effort to improve sampling techniques for obtaining accurate and inexpensive wilderness use estimates is clearly warranted. 


\section{LITERATURE CITED}

Cochran, William G.

1963. Sampling techniques. (Second edition) New York: John Wiley \& Sons.

413 p., illus.

Cushwa, Charles T., Burd S. McGinnes, and Thomas H. Ripley

1965. Forest recreation estimates and predictions in the North River area, George Washington National Forest, Virginia. Va. Polytech. Inst. Agr. Exp. Sta. Bul1. 558. Blacksburg, Va. 48 p., illus.

Hendee, John C., William R. Catton, Jr., Larry D. Marlow, and C. Frank Brockman

1968. Wilderness users in the Pacific Northwest - their characteristics, values, and management preferences. USDA Forest Serv. Res. Pap. PNW-61.

92 . . , illus.

James, George A., and Robert K. Henley

1968. Sampling procedures for estimating mass and dispersed recreation use on large areas. USDA Forest Serv. Res. Pap. SE-31. 15 p., illus.

Lucas, Robert C.

1964. Recreational use of the Quetico-Superior area. USDA Forest Serv. Res. Pap. LS-8. 50 p., i11us.

Thorse11, J. W.

1967. Wilderness recreational use: Waterton Lakes National Park. Recreational Res. Pap. 24. Nat. Park Serv., Dep. Indian Aff. and Northern Develop., Ottawa. 57 p., illus.

1968. Trail use survey: Banff and Yoho National Parks. Recreational Res. Rep. 33. Nat. Park Serv., Dep. Indian Aff. and Northern Develop., Ottawa. $57 \mathrm{p}$., illus.

University of California Wild1and Research Center

1962. Wilderness and recreation--a report on resources, values, and problems. ORRRC Study Rep. 3. Outdoor Recreation Resources Rev. Comm., Wash., D.C. 352 p., illus.

USDA Forest Service

1959. Nationa1 Forest Recreation Survey (NFRS). (Unpublished.) Wash., D.C. 123 p., i11us.

Wenger, Wiley D., Jr.

1964. A test of unmanned registration stations on wilderness trails: factors influencing effectiveness. USDA Forest Serv. Res. Pap. PNW-16. $48 \mathrm{p}$., illus.

, and H. M. Gregersen

1964. The effect of non-response on representativeness of wilderness-trail register information. USDA Forest Serv. Res. Pap. PNW-17. 20 p., illus. 


\section{APPENDIX I}

\section{Primitive Area Registration Card}

PRIMITIVE AREA REGISTRATION CARD

COMPLETE THIS CARD AND PLACE THIS PORTION IN SLOT IN BOX

Only ONE person from each group needs to complete the card. Please answer ALI questions.

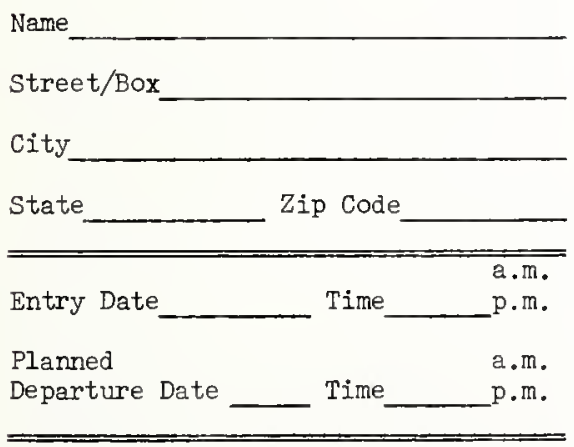

Number of persons in your group

Method of Travel: Hiking__ Riding_

No. of Saddle Animals

No. of Pack Animals

Is party with guide or outfitter?

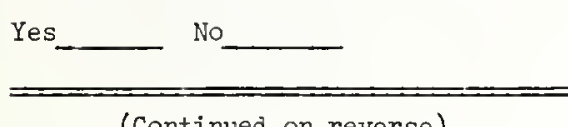

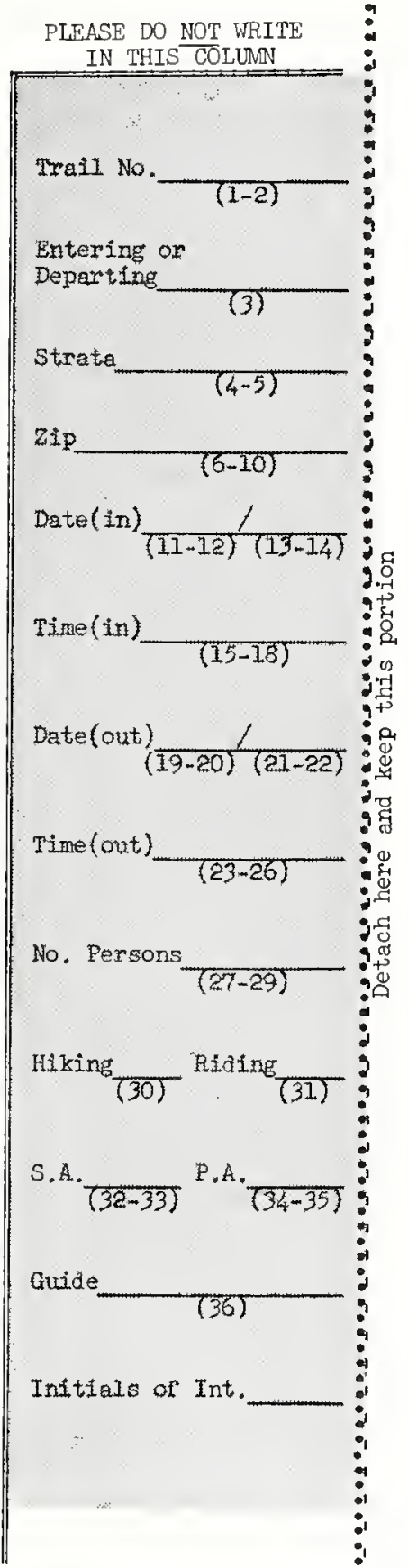

PRIMITIVE AREA REGISTRATION

U. S. Department of Agriculture Forest Service

- RETAIN THIS PORTION WHILE ON TRIP WELCOME TO

THE MISSION MOUNTAINS PRIMITIVE AREA

This Primitive Area is in a delicate state of natural balance. Any careless act by man may upset this balance and result in destruction of the primitive environment. You can help to retain its natural state by following these basic rules.

Do not smoke while traveling or throw litter along trails. Lead all pack stock-do not herd. Keep stock on trail where possible. Do not take shortcuts through meadows or across switchbacks.

Keep pack and saddle stock away from camp areas.

Stock should not be picketed or tied to trees overnight. Instead, use hobbles, natural barriers, and stock handling facilities where provided. Carry supplemental horse feed and avoid using the heavily grazed areas.

Figure 9. - Front side of the registration card used for the study. 
Refer to the attached map which you may keep for use on your trip. ENTER HERE the number or numbers of the compartments that you plan to visit on your trip.

If you are planning to stay overnight, name the places you plan to camp and the number of nights planned for each place.

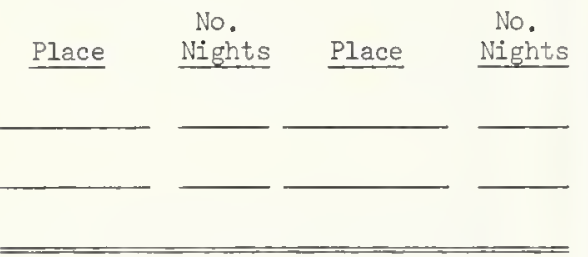

Which of the following activities do you (and your group) plan to engage in during your visit? State in each one how many of you plan to do the particular activity. Complete as many as needed.

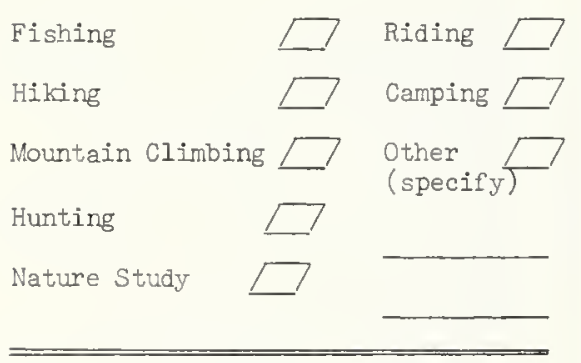

3.O.B. No. $40-568048 \quad 12 / 31 / 68$

USDA-Forest Service

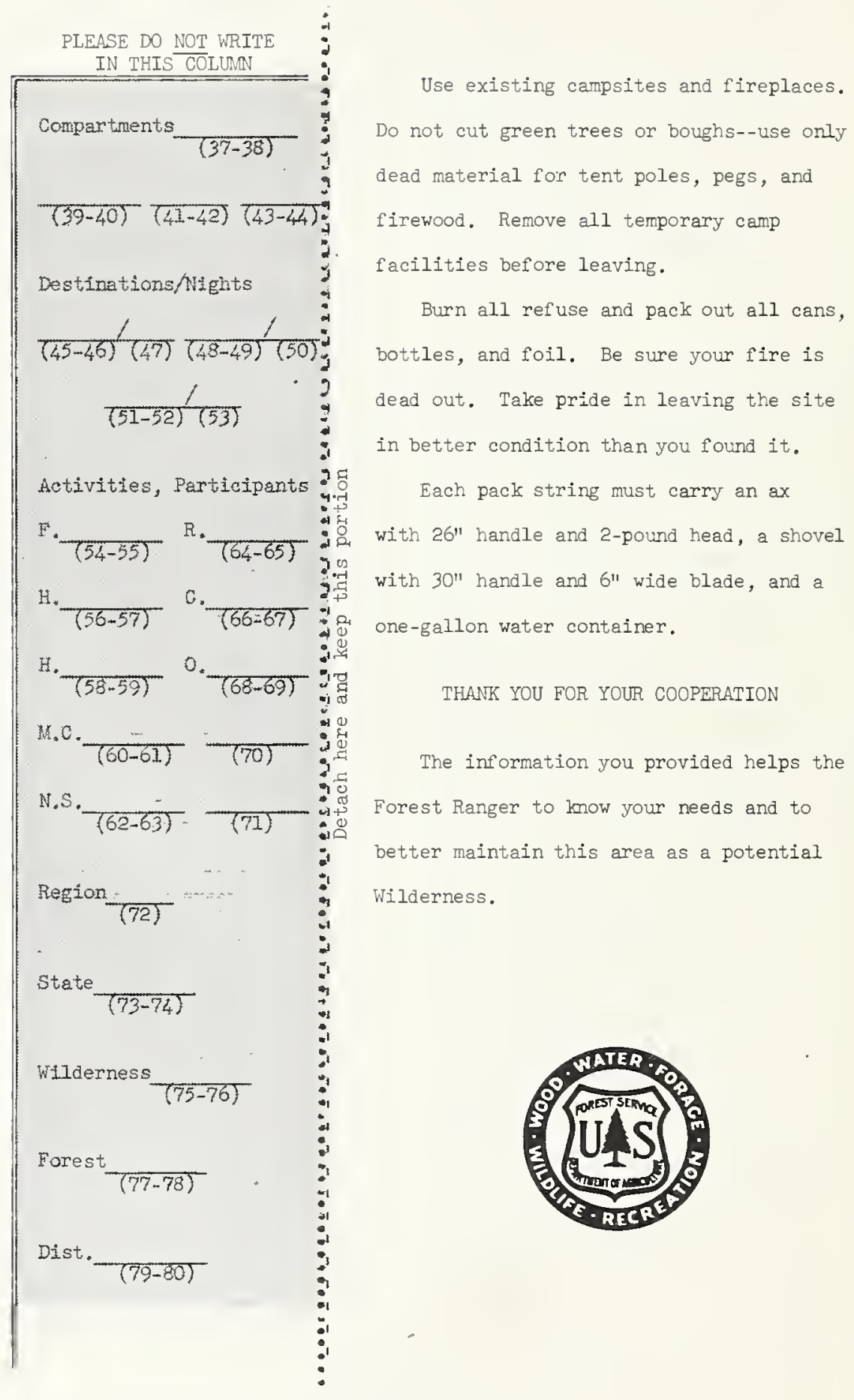

Figure 10. - Back side of the registration card used for the study. 


\section{APPENDIX II \\ Formula Used to Calculate Standard Errors for Estimated Registration Rates}

The formula (Cochran, 1963, section 6.11) used to derive standard error follows:

$$
\operatorname{var} \hat{Y}_{h}=R_{h}^{2} \frac{\left(1-\frac{r_{h}}{R_{h}}\right)}{r_{h}}\left[S^{2} m_{h}+\hat{Y}_{h}{ }^{2} S^{2} n_{h}-2 \hat{Y}_{h} S^{S} m_{h} S_{n_{h}}\right] / M_{h}{ }^{2}
$$

for individual strata, and for pooled estimates

$$
\operatorname{var}(\hat{Y})=\sum_{h=1}^{7} R_{h}^{2} \frac{\left(1-\frac{r}{R_{h}}\right)}{r_{h}}\left[S^{2} m_{h}+\hat{Y}_{h}{ }^{2} S^{2} n_{h}-2 \hat{Y} \hat{\rho}_{h} S_{m_{h}} S_{h}\right] / M_{h}{ }^{2} .
$$

To estimate weighted means for pooled strata, we used

$$
\hat{Y}=\frac{\sum_{h=1}^{7} \frac{R_{h}}{R} \sum_{i=1}^{r_{h}} \frac{m_{h i}}{r_{h}}}{\sum_{h=1} \frac{R_{h}}{R} \sum_{i=1}^{r_{h}} \frac{n_{h i}}{r_{h}}}
$$

where

$\hat{Y}_{h}=$ estimated registration rate in stratum $\mathrm{h} ; \hat{\mathrm{Y}}=$ overall estimated rate;

$\mathrm{R}_{\mathrm{h}}=$ actual total number of sample units in stratum $\mathrm{h}$;

$R=\sum_{h=1} R_{h}$, or the total number of sample units;

$r_{h}=$ the number of selected sample units in stratum $h$;

$M_{h}=$ the number of registered groups;

$m_{h i}=$ the number of registered sample groups from sample unit $i$, stratun $h$;

$\mathrm{n}_{\mathrm{hi}}=$ the total number of sample groups from sample unit $i$, stratum $\mathrm{h}$;

$\mathrm{S}^{2} \mathrm{~m}_{\mathrm{h}}=$ variance of $\mathrm{m}_{\mathrm{h}}$ (and similarly for $\mathrm{n}_{\mathrm{h}}$ );

$\hat{\rho}_{h}=$ estimated correlation of $m_{h i}$ and $n_{h i}$. 


\section{APPENDIX III \\ Variables and Notation Used in Study}

Variables and notation used in this study include:

$x_{i}$ : registration information for variable $i$.

$Y_{i}$ : interview information (entering) for variable $i$.

$z_{i}$ : interview information (leaving) for variable $i$.

Variables of interest include:

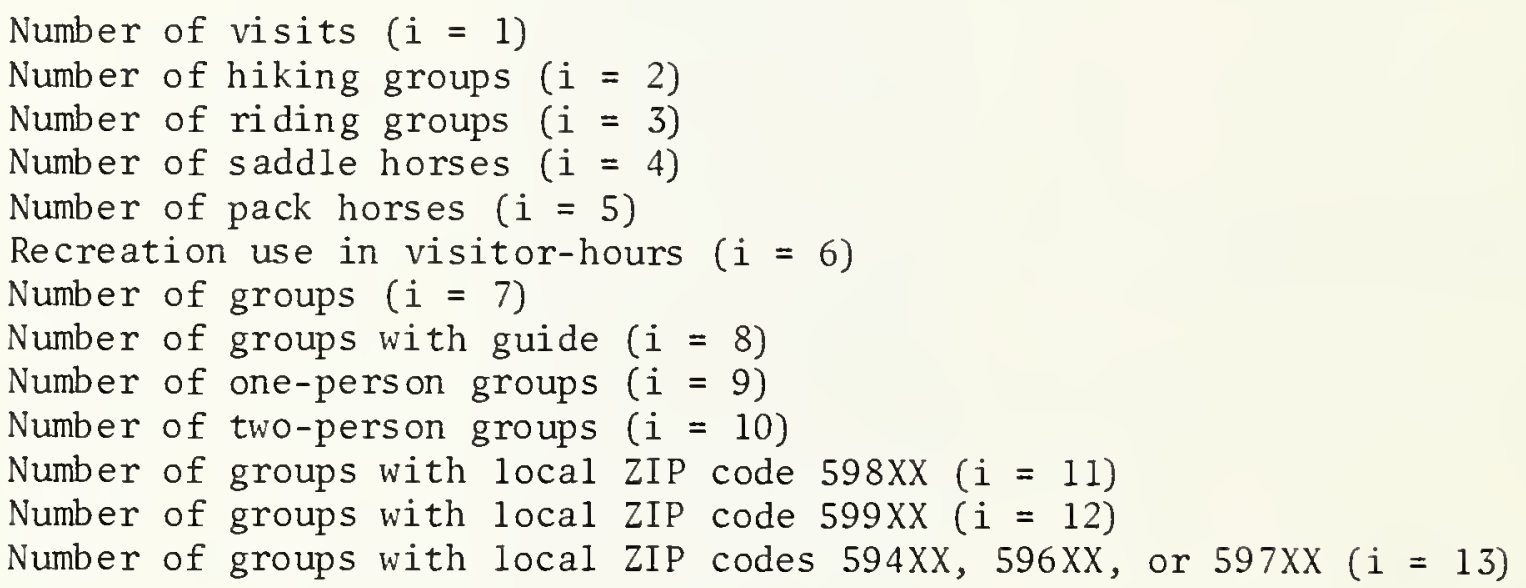

Table 10.--Totals from registration cards for 13 study variables, by stration.

\begin{tabular}{|c|c|c|c|c|c|c|c|c|c|c|}
\hline \multirow[t]{2}{*}{ Variable } & \multicolumn{10}{|c|}{ Stratum } \\
\hline & 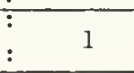 & $\begin{array}{l}: \\
:\end{array}$ & $\begin{array}{l}\vdots \\
\vdots\end{array}$ & : & $\begin{array}{l}\vdots \\
\vdots\end{array}$ & $\begin{array}{l}: \\
\vdots\end{array}$ & 7 & : Summer & : Fall & Total \\
\hline \multicolumn{11}{|l|}{ Number of: } \\
\hline Visits $\left(X_{1}\right)$ & 658 & 597 & 570 & 880 & 140 & 136 & 78 & 2,705 & 354 & 3,059 \\
\hline Hiking groups $\left(x_{2}\right)$ & 163 & 167 & 133 & 168 & 48 & 42 & 19 & 631 & 109 & 740 \\
\hline Riding groups $\left(x_{3}^{2}\right)$ & 3 & 2 & 4 & 40 & 3 & 11 & 7 & 49 & 21 & 70 \\
\hline Saddle animals $\left(x_{4}\right)$ & 6 & 0 & 16 & 172 & 6 & 34 & 11 & 194 & 51 & 245 \\
\hline Pack animals $\left(\mathrm{X}_{5}\right)$ & 0 & 0 & 0 & 65 & 1 & 7 & 1 & 65 & 9 & 74 \\
\hline Total use, hours $\left(\mathrm{x}_{6}\right)$ & 6,511 & 4,533 & 3,932 & 15,289 & 1,397 & 2,375 & 755 & 30,265 & 4,527 & 34,855 \\
\hline \multicolumn{11}{|l|}{ Number of: } \\
\hline Groups $\left(X_{7}\right)$ & 166 & 169 & 137 & 208 & 51 & 53 & 26 & 680 & 130 & 810 \\
\hline Groups with guides $\left(x_{8}\right)$ & 1 & 4 & 1 & 19 & 2 & 2 & 0 & 25 & 4 & 29 \\
\hline One-person groups $\left(x_{9}\right)$ & 7 & 20 & 7 & 19 & 3 & 6 & 4 & 53 & 13 & 66 \\
\hline Two-person groups $\left(x_{10}\right)$ & 40 & 50 & 39 & 62 & 21 & 29 & 12 & 191 & 62 & 253 \\
\hline $\begin{array}{l}\text { Groups with } 2 \text { IP code } \\
\left.598 \times X_{11}\right)\end{array}$ & 93 & 53 & 62 & 119 & 26 & 17 & 13 & 327 & 56 & 383 \\
\hline $\begin{array}{l}\text { Groups with ZIP code } \\
599 \times X\left(X_{12}\right)\end{array}$ & 20 & 24 & 31 & 16 & 17 & 23 & 9 & 91 & 49 & 140 \\
\hline $\begin{array}{l}\text { Groups with ZIP codes } \\
594 X X, 596 X X \text {, or } 597 X X \\
\left(X_{13}\right)\end{array}$ & 19 & 22 & 6 & 16 & 3 & 6 & 1 & 63 & 10 & 73 \\
\hline
\end{tabular}


Headquarters for the Intermountain Forest and Range Experiment Station are in Ogden, Utah. Field Research Work Units are maintained in:

Boise, Idaho

Bozeman, Montana (in cooperation with Montana State University)

Logan, Utah (in cooperation with Utah State University)

Missoula, Montana (in cooperation with University of Montana)

Moscow, Idaho (in cooperation with the University of Idaho)

Provo, Utah (in cooperation with Brigham Young University) 
